\title{
Biochemical components of wild relatives of chickpea confer resistance to pod borer, Helicoverpa armigera
}

\author{
Siva Kumar Golla ${ }^{1,2} \cdot$ Hari Chand Sharma ${ }^{1} \cdot$ P. Rajasekhar ${ }^{2} \cdot$ Suraj Prashad Mishra ${ }^{1} \cdot$ Jagdish Jaba $^{1}$
}

Received: 7 March 2020 / Accepted: 13 April 2020

(c) Springer Nature B.V. 2020

\begin{abstract}
Efforts are being made to develop chickpea varieties with resistance to the pod borer, Helicoverpa armigera for reducing pesticide use and minimizing the extent of losses due to this pest. However, only low to moderate levels of resistance have been observed in the cultivated chickpea to this polyphagous pest. Hence, it is important to explore wild relatives as resistance sources to develop insect-resistant cultivars. Therefore, we studied different biochemical components that confer resistance to H. armigera in a diverse array of wild relatives of chickpea. Accessions belonging to wild relatives of chickpea exhibited high levels of resistance to $H$. armigera as compared to cultivated chickpea genotypes in terms of lower larval survival, pupation and adult emergence, decreased larval and pupal weights, prolonged larval and pupal developmental periods and reduced fecundity of the H. armigera when reared on artificial diet impregnated with lyophilized leaf powders. Amounts of proteins and phenols in different accessions of chickpea wild relatives were significantly and negatively correlated with larval weight, pupation and adult emergence. Phenols showed a negative correlation with pupal weight and fecundity, but positive correlation with pupal period. Total soluble sugars showed a negative correlation with larval period, but positive correlation with pupation and pupal weight, while tannins showed a positive correlation with larval weight, pupation and adult emergence. The flavonoid compounds such as chlorogenic acid, ferulic acid, naringin, 3,4-dihydroxy flavones, quercetin, naringenin, genistein, biochanin-A and formononetin that were identified through HPLC fingerprints, exhibited negative effects on survival and development of $H$. armigera reared on artificial diet impregnated with lyophilized leaf powders. The wild relatives with diverse mechanisms of resistance conferred by different biochemical components can be used as sources of resistance in chickpea breeding programs to develop cultivars with durable resistance to $H$. armigera for sustainable crop production.
\end{abstract}

Keywords Chickpea $\cdot$ Wild relatives $\cdot$ Helicoverpa armigera $\cdot$ Antibiosis $\cdot$ Biochemical mechanism of resistance

\section{Introduction}

Chickpea (Cicer arietinum L.) ranks third among the pulse crops after dry beans and peas in terms of total production worldwide. Chickpea is grown in over 50 countries globally on an area of 12.65 million ha and production of 12.09 million tonnes, of which Asia alone accounts for 10.68 million ha area and 9.70 million tonnes production

Handling Editor: Heikki Hokkanen.

Siva Kumar Golla

siva77brinda@gmail.com

1 International Crops Research Institute for the Semi-Arid Tropics (ICRISAT), Patancheru, Telangana 502324, India

2 Acharya N.G, Ranga Agricultural University, Guntur, Andhra Pradesh 522509, India
(FAO STAT 2016). Legume pod borer, Helicoverpa armigera (Hubner) is the most important constraint for chickpea production worldwide. The early instar $H$. armigera larvae feed on leaves of chickpea, while later instars shift to flowers and developing pods and cancause $80-90 \%$ reduction in yield despite several rounds of insecticide applications (Banu et al. 2005). It is very difficult to control this pest due to its high fecundity, multiple generations, polyphagous feeding habit, and long distance migration (Sarode 1999) and ability to develop resistance to insecticides (Sharma 2001; Kranthi et al. 2002). Therefore, there is need for deploying alternative methods of controlling this pest, of which host plant resistance can provide an effective means to minimize the extent of crop losses. However, only moderate levels of resistance have been identified in the available cultivated chickpea germplasm, while the wild relatives of chickpea have shown high levels of 
resistance to H. armigera (Sharma et al. 2005a, b, 2006; Golla et al. 2018). Hence, there is a necessity to identify accessions with different components of resistance, to use in breeding programs to broaden the basis and increase the levels of resistance to this pest for sustainable crop production.

The wild relatives of chickpea that have shown high levels of resistance to $H$. armigera have not yet been characterized for biochemical mechanisms of resistance. Plant-herbivore interactions are the resultant of nutritional composition of the host plant, and the influence of morphological traits and secondary metabolites on the herbivores (Cates 1980). Primary and secondary metabolites of the host plant influence the insect behavior and their survival and development (Roeder and Behmer 2014). Secondary metabolites reduce the digestibility of plant tissues in the insect gut, and thereby affect the larval growth and development (Bennett and Wallsgrove 1994), whereas the effect of primary metabolites or nutritional factors depends on the relative amounts of different constituents (Behmer 2008). The suboptimal ratio between carbohydrates and proteins reduces the insect growth and development (Simpson and Raubenheimer 2009; Roeder and Behmer 2014).

Chickpeas are known for their inhibitory activity of gut proteinases (Saini et al. 1992; Patankar et al. 1999).Wild relatives of chickpea exhibit considerable diversity in protease inhibitor isoforms as compared to the cultivated chickpea (Patankar et al. 1999). Among the secondary metabolites, phenols play a significant role in conferring resistance to insect pests by adversely affecting larval growth and development by feeding inhibition and/or by reduced larval metabolism (Treutter 2006; Ballhorn et al. 2011). Development of plant with resistance to insect pests has often been attributed to high phenol content (Selvanarayanan and Narayanasamy 2006). Severity of the adverse effects of tannins in different insects ranges from no visual effect to reduction in growth and development, and finally mortality of the insect (Panzuto et al. 2002). However, the effects of tannins in insect gut depend on their concentration and chemical structure of the tannins, as well as $\mathrm{pH}$ and concentration of antioxidants in the insect gut (Galati et al. 2002; Hagerman et al. 2003). Flavonoid compounds are biosynthesized via the phenylpropanoid pathway (Dakora and Phillips 1996) in the host plant and affect insect feeding, survival, growth and fecundity (Musayimana et al. 2001; Napal et al. 2010). The flavonoid compounds such as quercetin, chlorogenic acid and rutin are widely distributed among crop plants and contribute to resistance against herbivores (Kennedy 2003; Simmonds 2003). Hence, a basic understanding of the interactions between the biochemical characters of wild relatives of chickpea and growth and development of $H$. armigera is highly important to identify biochemical constituents contributing to host plant resistance to this insect for use as selection criteria to develop chickpea cultivars with stable and high levels of resistance to this pest.

\section{Materials and methods}

\section{Chickpea genotypes}

Fifteen accessions of the wild relatives of chickpea belonging to seven species of Cicer, along with five accessions of cultivated chickpea (Cicer arietinum) were evaluated for resistance to pod borer, H. armigera. All the genotypes were grown under field conditions during post rainy seasons, 2014-2015 and 2015-2016 at the International Crops Research Institute for the Semi-Arid Tropics (ICRISAT), Patancheru, Telangana, India. Each accession was sown in a two row plot, and each row being $2 \mathrm{~m}$ long. There were two replications in a randomized complete block design. Seeds of the wild accessions were scarified at one end using scalpel to enhance water absorption, soaked in water for $24 \mathrm{~h}$, and treated with thiram ( $3 \mathrm{~g}$ per $\mathrm{kg}$ of seed) before sowing. The seeds of cultivated chickpea were sown without scarification. The experimental trial was laid out with a spacing of $60 \times 30 \mathrm{~cm}$ between the rows and plants in black soil (Vertisols), and the crop was raised under insecticide-free conditions.

In the glasshouse, all genotypes were planted in plastic pots $(30 \times 30 \mathrm{~cm})$ filled with a potting mixture of black soil, sand and farmyard manure (2:1:1) at ICRISAT, Patancheru, Telangana, India. Five seedlings were raised in each pot and there were three pots for each accession in a completely randomized design. The plants in pots were watered as and when necessary. Desert coolers were used to maintain the temperature of $27 \pm 5{ }^{\circ} \mathrm{C}$, and relative humidity of $>65 \%$ in the glasshouse.

\section{Helicoverpa armigera culture}

The neonates of $H$. armigera used in bioassays were obtained from the laboratory reared culture atthe International Crops Research Institute for the Semi-Arid Tropics (ICRISAT), Patancheru, Telangana State, India. Under laboratory conditions, the $H$. armigera larvae were reared individually on chickpea-based artificial diet (Babu et al. 2014) at $27 \pm 2{ }^{\circ} \mathrm{C}$ temperature, 65 to $75 \%$ relative humidity, and $16: 8 \mathrm{~h}(\mathrm{~L} / \mathrm{D})$ photoperiod regime.

\section{Survival and development of $\boldsymbol{H}$. armigera on different wild relatives of chickpea}

The antibiosis component of resistance to $H$. armigera in the wild relatives of chickpea was evaluated by rearing the neonate larvae on artificial diet impregnated with lyophilized 
leaf powders of different chickpea genotypes (Narayanamma et al. 2008). The chickpea terminals or branches with tender green leaves were collected from the plants grown in the field and the glasshouse at the full vegetative growth stage, and placed in an icebox and eventually frozen at $-20{ }^{\circ} \mathrm{C}$ (REMI, Model-RQF 425, Japan). The leaves were freezedriedto avoid changes in biochemical composition using lyophilizer (Modulyo D, Thermo Savant, Japan) at $-45^{\circ} \mathrm{C}$ temperature and 436 mbar pressure for 3 to 4 days. The leaves were powdered and stored in a desiccator till used. Dried powder of chickpea leaves $(20 \mathrm{~g})$ was incorporated into the artificial diet as a replacement for part of the chickpea flour for rearing of $H$. armigera. The neonate larvae of $H$. armigera were released individually on the diet in a 25 cell-well plate with a fine camel hairbrush. Each treatment was replicated thrice (25 larvae in each replication) in completely randomized design. The cell-wells were maintained at $27 \pm 2{ }^{\circ} \mathrm{C}$ temperature, 65 to $75 \%$ relative humidity and $12 \mathrm{~h}$ photoperiod after releasing the neonates onto the artificial diet. Data were recorded on larval survival, and the larval weights on 10th day after releasing the larvae into artificial diet. Pupal weights were recorded one day after pupation. Pupae from each replication were sterilized with $2 \%$ sodium hypochlorite solution and placed in a plastic jar containing moist vermiculite. Data were also recorded on larval and pupal periods. The adults were collected from the jars, and three pairs of adults that emerged on the same day on a particular genotype were placed inside a plastic cage, and the numbers of eggs laid were counted. Percentage of larval survival on 10th day, pupation and adult emergence were computed in relation to number of neonate larvae released in each replication.

\section{Biochemical characterization of different wild relatives of chickpea}

Biochemical components in the lyophilized leaf powder of different genotypes of chickpea were estimated using standard protocols. Protein content was estimated as per Lowry et al. (1951), phenol content as per the method presented by Bray and Thorpe (1954), tannin content by vanillin hydrochloride method (Burns 1971), and total soluble sugar content as per Hedge and Hofreiter (1962).

\section{Estimation of flavonoids in wild relatives of chickpea through HPLC fingerprints}

Flavonoid compounds from different wild relatives of chickpea were extracted by the method described by Hahn et al. (1983) with slight modifications. Lyophilized leaf sample $(100 \mathrm{mg}$ ) was homogenized in $5 \mathrm{ml}$ of HPLC grade methanol with mortar and pestle and centrifuged at $8000 \mathrm{rpm}$ for $20 \mathrm{~min}$. The supernatant was collected and partitioned with three times the volume of hexane in a separation funnel, and the methanol phase was collected. This process was repeated three times. Collected methanol phase was concentrated to a volume of $2 \mathrm{ml}$ in a vacuum rotavapor (R-215, Buchi, Switzerland). Concentrated samples were filtered through $0.22 \mu \mathrm{m}$ millipore filter and injected into HPLC (High Performance Liquid Chromatography). The standards such as chlorogenic acid, ferulic acid, naringin, 3,4-dihydroxy flavones, quercetin, naringenin, genistein, formononetin and biochanin $A$ were prepared at $1000 \mathrm{ppm}$ concentrations, and filtered as described above.

Flavonoid compounds in different samples were chromatographed using HPLC equipped with Sunfire $\mathrm{C}_{18}$ column $(4.6 \times 250 \mathrm{~mm})$ with $5 \mu \mathrm{m}$ pore size, and Waters 2695 separations module system consisting of a PCM 11 reciprocating piston pump. The sample retention time was recorded with photodiode array detector (Waters 2996). Multistep gradient solvent system was used for separation of compounds which consisted of $2 \%$ acetic acid in Millipore water (Solvent A) and $2 \%$ acetic acid in acetonitrile (Solvent $B$ ). The separation was programmed isocratically: $5 \%$ of solvent $B$ for $10 \mathrm{~min}$, followed by a 7.5 min linear gradient to $15 \%$ of solvent B, which was run isocratically for $13.5 \mathrm{~min}$, followed by a $10 \mathrm{~min}$ linear gradient to $50 \%$ of solvent $\mathrm{B}$. This was run isocratically for 4 min, followed by a 5 min linear gradient to $15 \%$ of solvent B, and finally followed by a 5 min linear gradient to $5 \%$ of solvent B. Flow rate was $1 \mathrm{ml} / \mathrm{min}$. Three replications were maintained for each genotype. The chromatographic data were recorded and processed by the Empower ${ }^{\mathrm{TM}}$. The flavonoid compounds were identified by retention times of the peaks calibrated with standards and quantification was done by comparing the peak area of the sample with peak area of the standard obtained with known concentrations at similar retention times.

\section{Statistical analysis}

The data were subjected to analysis of variance (ANOVA) by using GENSTAT 14.0 version. The significance of differences between treatments was measured by $F$-test and the treatment means were compared by least significant difference (LSD) at $P=0.05$. The data on survival and development of $H$. armigera on artificial diet with lyophilized leaf powders and biochemical characters of different genotypes were subjected to similarity matrix analysis with nearest neighbors to assess the diversity among wild relatives of chickpea with resistance to the pod borer, H. armigera. 


\section{Results}

\section{Survival and development of $\boldsymbol{H}$. armigera on artificial diets with lyophilized leaf powder of wild relatives of chickpea grown under field conditions}

\section{Post-rainy season, 2014-2015}

Significant differences were observed in survival and development of $H$. armigera when the neonates were reared on artificial diet impregnated with lyophilized leaf powder of different genotypes during the post-rainy season, 2014-2015 (Table 1). On 10th day after releasing the larvae on to the diet, lowest larval survival was recorded on the resistant check, ICC 506 EB (58.33\%), which was not significantly different from IG 599076 (60.42\%), ICCL 86111 (60.42\%), PI 599066 (60.42\%), IG 70012 (62.50\%) and PI 599046 (62.50\%). Highest larval survival (87.50\%) was recorded on IG 72933, and the susceptible check, ICC 3137. Larval weight on 10th day was significantly lower on all the wild relatives as compared to that on JG 11 (19.94 mg) and the susceptible check, KAK 2 (17.46 mg). The lowest larval weights were recorded in insects reared on IG $69,979(2.55 \mathrm{mg})$. Larval period of $H$. armigera was prolonged significantly in insects reared on the wild relatives of chickpea ( $>25.0$ days) as compared to the larvae fed on the susceptible check, ICC 3137 (23.52 days).

Pupation was significantly lower (27.08\%) in $\mathrm{H}$. armigera larvae reared on C. bijugum (IG 70012, IG 70018 and PI 599046), while highest pupation was observed in insects reared on JG 11 (54.17\%). The weights of $H$. armigera pupae were significantly lower in insects reared on the wild relatives of chickpea as compared to the larvae reared on the susceptible check, ICC 3137 (417.27 mg), but not significantly different from the larvae reared on other cultivated chickpea. Longest pupal period was observed in insects reared on IG 70018 (15.82 days), followed by IG 70022 (15.41 days), PI 599066 (15.39 days) and PI 510663 (15.35 days), while the shortest pupal period was observed in insects reared on the susceptible checks, KAK 2 (12.17 days) and ICC 3137 (12.43 days). Adult emergence of $H$. armigera when reared on all the wild relatives was observed in a range of $16.67 \%$ (IG 70018 and PI 599046) and 33.33\% (IG 72953, PI 510663, PI 568217 and PI 599077) and significantly lowest compared to susceptible checks, KAK 2 (47.92\%) and ICC 3137 (45.83\%). Fecundity of $H$. armigera females was lowest when the larvae were reared on PI 599066 (214.42 eggs/ female), and highest in larvae reared on JG 11 (389.42 eggs/ female), the later was not significantly different fromthose reared on the susceptible checks, ICC 3137 (349.25 eggs/ female) and KAK 2 (340.17 eggs/female).
Post-rainy season, 2015-2016

Survival and development of $H$. armigera on artificial diet impregnated with lyophilized leaf powders was significantly different among the genotypes tested during post-rainy season, 2015-2016 (Table 2). Larval survival of $H$. armigera on 10th day was significantly lowest on IG 70006 (50.00\%), followed by IG 70012 (58.33\%) and IG 72933 (58.33\%). Highest larval survival was observed on the susceptible check, KAK 2 (91.67\%), which was not significantly different from JG 11 (89.58\%), ICC 3137 (87.50\%) and PI 599077 (87.50\%). Significantly lower larval weights were recorded in larvae reared on artificial diets with leaf powder of the wild relatives of chickpea (3.61 mg on IG 70018 to $11.24 \mathrm{mg}$ on IG 72953) as compared to the larvae reared on diets with leaf powder of JG $11(17.12 \mathrm{mg})$ and the susceptible check, KAK $2(16.19 \mathrm{mg})$. Larval period of $H$. armigera was prolonged by 2-3 days when the larvae were reared on wild relatives of chickpea as compared to that on the susceptible check, ICC 3137 (22.35 days). Longest larval period was observed in larvae reared on diets with leaf powder of C. microphyllum accession, ICCW 17148 (26.94 days), followed by C. bijugum, IG 70018 (26.77 days).

Significantly lower pupation was observed in larvae reared on diets with leaf powder of wild relatives of chickpea as compared to the larvae reared on diets with leaf powder of susceptible check, KAK 2 (72.92\%), except on PI 599077 (64.58\%) and PI 599109 (58.33\%). Mean pupal weights were lower in insects reared on diets with leaf powder of wild relatives of chickpea (321.68 mg on IG 70012 to $410.63 \mathrm{mg}$ on IG 72953) as compared to the larvae reared on diets with leaf powder of JG 11 (464.73 mg), which was not significantly different from susceptible checks, ICC 3137 (446.31 mg) and KAK 2 (427.42 mg). Pupal period of was significantly longer in larvae reared on diets with leaf powder of IG 70012 (15.81 days) and IG 70018 (15.73 days) as compared to the larvae reared on diets with leaf powder of JG 11 (11.63 days) and ICC 3137 (11.77 days). Adult emergence on the wild genotypes ranged $12.50 \%$ (IG 70006) to $39.58 \%$ (PI 599077 and ICCW 17148), which was significantly lower than on JG 11 (56.25\%), ICC 3137 (54.17\%) and KAK 2 (50.00\%). Lowest fecundity was observed when the insects were reared on diets with leaf powder of IG 70018 (207.33 eggs/female), followed by IG 70012 (211.33 eggs/female), while highest fecundity was recorded in insects reared on JG 11 (382.33 eggs/female) and ICC 3137 (382.00 eggs/female).

\section{Survival and development of $\boldsymbol{H}$. armigera on artificial diets with lyophilized leaf powder of wild relatives of chickpea grown under glasshouse conditions}

Survival and development of $H$. armigera on artificial diet impregnated with lyophilized leaf powders varied 
Table 1 Expression of resistance to $H$. armigera in wild relatives of chickpea grown under field conditions (diet incorporation assay, post-rainy season, 2014-2015)

\begin{tabular}{|c|c|c|c|c|c|c|c|c|c|}
\hline Species & Genotype & $\begin{array}{l}\text { Larval } \\
\text { survival } 10 \\
\text { DAE }(\%)^{\#}\end{array}$ & $\begin{array}{l}\text { Mean larval } \\
\text { weight } 10 \\
\text { DAE }(\mathrm{mg})\end{array}$ & $\begin{array}{l}\text { Larval } \\
\text { period } \\
\text { (days) }\end{array}$ & $\begin{array}{l}\text { Pupation } \\
(\%)^{\#}\end{array}$ & $\begin{array}{l}\text { Mean pupal } \\
\text { weight (mg) }\end{array}$ & $\begin{array}{l}\text { Pupal } \\
\text { period } \\
\text { (days) }\end{array}$ & $\begin{array}{l}\text { Adult emer- } \\
\text { gence }(\%)\end{array}$ & $\begin{array}{l}\text { Fecundity } \\
\text { (eggs/ } \\
\text { female) }\end{array}$ \\
\hline $\begin{array}{l}\text { C. chros- } \\
\text { sanicum }\end{array}$ & IG 599076 & $\begin{array}{l}60.42 \\
\quad(51.02)^{\mathrm{ab}}\end{array}$ & $4.34^{\mathrm{ab}}$ & $26.13^{\text {cde }}$ & $\begin{array}{l}31.25 \\
(33.98)^{\mathrm{a}}\end{array}$ & $354.11^{\mathrm{ab}}$ & $14.30^{\text {bcde }}$ & $\begin{array}{l}29.17 \\
(32.63)^{\text {abcde }}\end{array}$ & $\begin{array}{l}273.92 \\
(16.56)^{\text {bcdef }}\end{array}$ \\
\hline $\begin{array}{l}\text { C. cunea- } \\
\text { tum }\end{array}$ & IG 69979 & $\begin{array}{l}83.33 \\
(67.77)^{\mathrm{cd}}\end{array}$ & $2.55^{\mathrm{a}}$ & $25.46^{\mathrm{abcde}}$ & $\begin{array}{l}33.33 \\
(35.22)^{\mathrm{ab}}\end{array}$ & $356.76^{\mathrm{ab}}$ & $14.02^{\text {abcde }}$ & $\begin{array}{l}31.25 \\
(33.98)^{\text {abcdef }}\end{array}$ & $\begin{array}{l}233.25 \\
\quad(15.29)^{\mathrm{ab}}\end{array}$ \\
\hline C. bijugum & IG 70006 & $\begin{array}{l}77.08 \\
(62.02)^{\mathrm{abcd}}\end{array}$ & $4.40^{\mathrm{ab}}$ & $25.34^{\mathrm{abcde}}$ & $\begin{array}{l}35.42 \\
(36.51)^{\mathrm{abc}}\end{array}$ & $355.05^{\mathrm{ab}}$ & $14.44^{\text {cde }}$ & $\begin{array}{l}18.75 \\
\quad(25.35)^{\mathrm{ab}}\end{array}$ & $\begin{array}{l}252.00 \\
(15.89)^{\mathrm{abcd}}\end{array}$ \\
\hline C. bijugum & IG 70012 & $\begin{array}{l}62.50 \\
(52.27)^{\mathrm{abc}}\end{array}$ & $2.90^{\mathrm{a}}$ & $25.70^{\text {cde }}$ & $\begin{array}{l}27.08 \\
(31.34)^{\mathrm{a}}\end{array}$ & $336.74^{\mathrm{a}}$ & $14.49^{\text {cde }}$ & $\begin{array}{l}18.75 \\
\quad(25.63)^{\mathrm{abc}}\end{array}$ & $\begin{array}{l}226.42 \\
(15.06)^{\mathrm{a}}\end{array}$ \\
\hline C. bijugum & IG 70018 & $\begin{array}{l}70.83 \\
(57.54)^{\mathrm{abcd}}\end{array}$ & $3.91^{\mathrm{ab}}$ & $26.66^{\mathrm{e}}$ & $\begin{array}{l}27.08 \\
(31.34)^{\mathrm{a}}\end{array}$ & $328.60^{\mathrm{a}}$ & $15.82^{\mathrm{e}}$ & $\begin{array}{l}16.67 \\
(23.93)^{\mathrm{a}}\end{array}$ & $\begin{array}{l}222.42 \\
(14.92)^{\mathrm{a}}\end{array}$ \\
\hline C. bijugum & IG 70022 & $\begin{array}{l}77.08 \\
(61.42)^{\mathrm{abcd}}\end{array}$ & $6.77^{\mathrm{abc}}$ & $26.69^{\mathrm{e}}$ & $\begin{array}{l}31.25 \\
(33.98)^{\mathrm{a}}\end{array}$ & $351.03^{\mathrm{ab}}$ & $15.41^{\mathrm{de}}$ & $\begin{array}{l}22.92 \\
(28.58)^{\mathrm{abcd}}\end{array}$ & $\begin{array}{l}275.17 \\
(16.60)^{\text {bcdef }}\end{array}$ \\
\hline $\begin{array}{l}\text { C. reticula- } \\
\text { tum }\end{array}$ & IG 72933 & $\begin{array}{l}87.50 \\
(70.53)^{\mathrm{d}}\end{array}$ & $10.31^{\mathrm{cd}}$ & $25.37^{\text {abcde }}$ & $\begin{array}{l}35.42 \\
(36.51)^{\mathrm{abc}}\end{array}$ & $372.91^{\mathrm{abc}}$ & $13.26^{\mathrm{abc}}$ & $\begin{array}{l}25.00 \\
(29.92)^{\mathrm{abcd}}\end{array}$ & $\begin{array}{l}326.25 \\
(18.07)^{\text {ghi }}\end{array}$ \\
\hline $\begin{array}{l}\text { C. reticula- } \\
\text { tum }\end{array}$ & IG 72953 & $\begin{array}{l}77.08 \\
(61.42)^{\mathrm{abcd}}\end{array}$ & $7.60^{\mathrm{bc}}$ & $25.26^{\mathrm{abcde}}$ & $\begin{array}{l}39.58 \\
(38.94)^{\mathrm{abcd}}\end{array}$ & $382.23^{\mathrm{abc}}$ & $13.50^{\mathrm{abcd}}$ & $\begin{array}{l}33.33 \\
(35.22)^{\text {bcdef }}\end{array}$ & $\begin{array}{l}343.25 \\
(18.54)^{\mathrm{ij}}\end{array}$ \\
\hline $\begin{array}{l}\text { C. pinnatifi- } \\
\text { dum }\end{array}$ & PI 510663 & $\begin{array}{l}72.92 \\
(58.79)^{\mathrm{abcd}}\end{array}$ & $3.58^{\mathrm{ab}}$ & $26.12^{\text {cde }}$ & $\begin{array}{l}41.67 \\
\quad(40.13)^{\mathrm{abcd}}\end{array}$ & $354.28^{\mathrm{ab}}$ & $15.35^{\mathrm{de}}$ & $\begin{array}{l}33.33 \\
(35.26)^{\text {bcdef }}\end{array}$ & $\begin{array}{l}285.75 \\
(16.92)^{\text {cdefg }}\end{array}$ \\
\hline C. judaicum & PI 568217 & $\begin{array}{l}85.42 \\
(68.03)^{\mathrm{cd}}\end{array}$ & $5.69^{\mathrm{ab}}$ & $26.13^{\text {cde }}$ & $\begin{array}{l}39.58 \\
(38.98)^{\mathrm{abcd}}\end{array}$ & $363.73^{\mathrm{abc}}$ & $13.35^{\mathrm{abc}}$ & $\begin{array}{l}33.33 \\
(35.22)^{\text {bcdef }}\end{array}$ & $\begin{array}{l}215.50 \\
(14.69)^{\mathrm{a}}\end{array}$ \\
\hline C. bijugum & PI 599046 & $\begin{array}{l}62.50 \\
(52.35)^{\mathrm{abc}}\end{array}$ & $3.36^{\mathrm{ab}}$ & $25.73^{\text {cde }}$ & $\begin{array}{l}27.08 \\
(31.21)^{\mathrm{a}}\end{array}$ & $341.76^{\mathrm{a}}$ & $14.22^{\mathrm{bcde}}$ & $\begin{array}{l}16.67 \\
(23.93)^{\mathrm{a}}\end{array}$ & $\begin{array}{l}216.00 \\
(14.71)^{\mathrm{a}}\end{array}$ \\
\hline C. bijugum & PI 599066 & $\begin{array}{l}60.42 \\
\quad(51.02)^{\mathrm{ab}}\end{array}$ & $3.19^{\mathrm{a}}$ & $25.67^{\text {bcde }}$ & $\begin{array}{l}31.25 \\
(33.88)^{\mathrm{a}}\end{array}$ & $344.54^{\mathrm{a}}$ & $15.39^{\mathrm{de}}$ & $\begin{array}{l}22.92 \\
(28.58)^{\mathrm{abcd}}\end{array}$ & $\begin{array}{l}214.42 \\
(14.66)^{\mathrm{a}}\end{array}$ \\
\hline C. judaicum & PI 599077 & $\begin{array}{l}75.00 \\
(60.00)^{\mathrm{abcd}}\end{array}$ & $4.17^{\mathrm{ab}}$ & $26.37^{\mathrm{de}}$ & $\begin{array}{l}43.75 \\
\quad(41.38)^{\mathrm{abcd}}\end{array}$ & $352.38^{\mathrm{ab}}$ & $14.59^{\text {cde }}$ & $\begin{array}{l}33.33 \\
(35.22)^{\text {bcdef }}\end{array}$ & $\begin{array}{l}240.25 \\
(15.51)^{\mathrm{abc}}\end{array}$ \\
\hline $\begin{array}{l}\text { C. pinnatifi- } \\
\text { dum }\end{array}$ & PI 599109 & $\begin{array}{l}75.00 \\
(60.00)^{\mathrm{abcd}}\end{array}$ & $4.43^{\mathrm{ab}}$ & $25.00^{\mathrm{abcde}}$ & $\begin{array}{l}39.58 \\
(38.94)^{\mathrm{abcd}}\end{array}$ & $353.35^{\mathrm{ab}}$ & $14.05^{\text {abcde }}$ & $\begin{array}{l}27.08 \\
(31.21)^{\mathrm{abcd}}\end{array}$ & $\begin{array}{l}256.92 \\
\quad(16.04)^{\text {abcde }}\end{array}$ \\
\hline $\begin{array}{l}\text { C. micro- } \\
\text { phyllum }\end{array}$ & $\begin{array}{l}\text { ICCW } \\
17148\end{array}$ & $\begin{array}{l}83.33 \\
(65.91)^{\mathrm{bcd}}\end{array}$ & $4.57^{\mathrm{ab}}$ & $25.84^{\text {cde }}$ & $\begin{array}{l}31.25 \\
(33.68)^{\mathrm{a}}\end{array}$ & $326.81^{\mathrm{a}}$ & $14.71^{\text {cde }}$ & $\begin{array}{l}25.00 \\
(29.23)^{\mathrm{abcd}}\end{array}$ & $\begin{array}{l}305.67 \\
(17.50)^{\text {fghi }}\end{array}$ \\
\hline $\begin{array}{l}\text { C. arieti- } \\
\text { num }\end{array}$ & JG $11(\mathrm{C})$ & $\begin{array}{l}85.42 \\
\quad(67.60)^{\mathrm{cd}}\end{array}$ & $19.94^{\mathrm{e}}$ & $23.65^{\mathrm{ab}}$ & $\begin{array}{l}54.17 \\
\quad(47.42)^{\mathrm{d}}\end{array}$ & $413.76^{\mathrm{c}}$ & $12.28^{\mathrm{a}}$ & $\begin{array}{l}45.83 \\
\quad(42.58)^{\mathrm{ef}}\end{array}$ & $\begin{array}{l}389.42 \\
(19.75)^{\mathrm{j}}\end{array}$ \\
\hline $\begin{array}{l}\text { C. arieti- } \\
\text { num }\end{array}$ & KAK 2 (S) & $\begin{array}{l}75.00 \\
\quad(60.00)^{\mathrm{abcd}}\end{array}$ & $17.46^{\mathrm{e}}$ & $24.45^{\mathrm{abcd}}$ & $\begin{array}{l}52.08 \\
\quad(46.22)^{\mathrm{cd}}\end{array}$ & $403.73^{\mathrm{bc}}$ & $12.17^{\mathrm{a}}$ & $47.92(43.80)^{\mathrm{f}}$ & $\begin{array}{l}340.17 \\
(18.45)^{\mathrm{hij}}\end{array}$ \\
\hline $\begin{array}{l}\text { C. arieti- } \\
\text { num }\end{array}$ & $\begin{array}{l}\text { ICC } 3137 \\
\text { (S) }\end{array}$ & $\begin{array}{l}87.50 \\
(69.56)^{d}\end{array}$ & $16.03^{\mathrm{e}}$ & $23.52^{\mathrm{a}}$ & $\begin{array}{l}50.00 \\
(45.00)^{\mathrm{bcd}}\end{array}$ & $417.27^{c}$ & $12.43^{\mathrm{ab}}$ & $\begin{array}{l}45.83 \\
(42.58)^{\mathrm{ef}}\end{array}$ & $\begin{array}{l}349.25 \\
(18.68)^{\mathrm{ij}}\end{array}$ \\
\hline $\begin{array}{l}\text { C. arieti- } \\
\text { num }\end{array}$ & $\begin{array}{l}\text { ICCL } \\
86111(\mathrm{R})\end{array}$ & $\begin{array}{l}60.42 \\
\quad(51.02)^{\mathrm{ab}}\end{array}$ & $10.73^{\text {cd }}$ & $24.16^{\mathrm{abc}}$ & $\begin{array}{l}43.75 \\
\quad(41.38)^{\mathrm{abcd}}\end{array}$ & $379.09^{\mathrm{abc}}$ & $13.06^{\mathrm{abc}}$ & $\begin{array}{l}35.42 \\
(36.51)^{\text {cdef }}\end{array}$ & $\begin{array}{l}306.75 \\
(17.50)^{\text {efghi }}\end{array}$ \\
\hline \multirow[t]{5}{*}{$\begin{array}{l}\text { C. arieti- } \\
\text { num }\end{array}$} & $\begin{array}{l}\text { ICC 506EB } \\
\text { (R) }\end{array}$ & $\begin{array}{l}58.33 \\
(49.96)^{\mathrm{a}}\end{array}$ & $11.93^{\mathrm{d}}$ & $24.30^{\mathrm{abc}}$ & $\begin{array}{l}41.67 \\
\quad(40.19)^{\mathrm{abcd}}\end{array}$ & $381.13^{\mathrm{abc}}$ & $13.06^{\mathrm{abc}}$ & $\begin{array}{l}37.50 \\
(37.730)^{\mathrm{def}}\end{array}$ & $\begin{array}{l}291.00 \\
(17.07)^{\operatorname{defgh}}\end{array}$ \\
\hline & $\mathrm{Fp}$ & 0.04 & $<.001$ & 0.03 & 0.02 & 0.02 & 0.003 & 0.004 & $<.001$ \\
\hline & Mean & 59.91 & 7.39 & 25.38 & 37.81 & 363.46 & 13.99 & 32.86 & 16.62 \\
\hline & $\mathrm{SE} \pm$ & 4.60 & 1.26 & 0.59 & 3.01 & 16.26 & 0.56 & 3.21 & 0.44 \\
\hline & $\begin{array}{l}\text { LSD } \\
\qquad(P=0.05)\end{array}$ & 13.61 & 3.73 & 1.75 & 8.92 & 48.12 & 1.66 & 9.50 & 1.30 \\
\hline
\end{tabular}

$C$ commercial cultivar, $S$ susceptible check, $R$ resistant check

The values followed by same alphabet did not differ significantly at $P \leq 0.05$ (DMRT)

\#Figures in the parentheses are Angular transformed values; $D A E$ days after initiation of experiment

${ }^{\# \#}$ Figures in the parentheses are square root $(\sqrt{ } x+0.5)$ transformed values 
Table 2 Expression of resistance to $H$. armigera in wild relatives of chickpea grown under field condition (diet incorporation assay, post-rainy season, 2015-2016)

\begin{tabular}{|c|c|c|c|c|c|c|c|c|c|}
\hline Species & Genotype & $\begin{array}{l}\text { Larval } \\
\text { survival } 10 \\
\text { DAE }(\%)^{\#}\end{array}$ & $\begin{array}{l}\text { Mean larval } \\
\text { weight } 10 \\
\text { DAE (mg) }\end{array}$ & $\begin{array}{l}\text { Larval } \\
\text { period } \\
\text { (days) }\end{array}$ & $\begin{array}{l}\text { Pupation } \\
(\%)\end{array}$ & $\begin{array}{l}\text { Mean pupal } \\
\text { weight (mg) }\end{array}$ & $\begin{array}{l}\text { Pupal } \\
\text { period } \\
\text { (days) }\end{array}$ & $\begin{array}{l}\text { Adult emer- } \\
\text { gence }(\%)\end{array}$ & $\begin{array}{l}\text { Fecundity } \\
\text { (eggs/ } \\
\text { female) }\end{array}$ \\
\hline $\begin{array}{l}\text { C. chros- } \\
\text { sanicum }\end{array}$ & IG 599076 & $\begin{array}{l}75.00 \\
(60.08)^{\text {bcde }}\end{array}$ & $4.26^{\mathrm{ab}}$ & $26.43^{\text {ghij }}$ & $39.58^{\mathrm{ab}}$ & $346.02^{\mathrm{abc}}$ & $13.82^{\text {cdefghi }}$ & $\begin{array}{l}27.08 \\
(31.21)^{\mathrm{de}}\end{array}$ & $\begin{array}{l}265.33 \\
(16.29)^{\text {bcdef }}\end{array}$ \\
\hline C. cuneatum & IG 69979 & $\begin{array}{l}79.17 \\
(62.95)^{\text {cdef }}\end{array}$ & $4.43^{\mathrm{ab}}$ & $25.52^{\text {efghi }}$ & $43.75^{\mathrm{abcd}}$ & $360.63^{\text {abcde }}$ & $14.14^{\text {defghij }}$ & $\begin{array}{l}35.42 \\
(36.45)^{\mathrm{fg}}\end{array}$ & $\begin{array}{l}230.00 \\
(15.17)^{\mathrm{abcd}}\end{array}$ \\
\hline C. bijugum & IG 70006 & $\begin{array}{l}50.00 \\
(45.00)^{\mathrm{a}}\end{array}$ & $5.81^{\text {abcde }}$ & $24.19^{\text {bcde }}$ & $41.67^{\mathrm{abc}}$ & $353.91^{\text {abcde }}$ & $14.15^{\text {defghij }}$ & $\begin{array}{l}12.50 \\
(20.70)^{\mathrm{a}}\end{array}$ & $\begin{array}{l}255.00 \\
(15.93)^{\text {abcde }}\end{array}$ \\
\hline C. bijugum & IG 70012 & $\begin{array}{l}58.33 \\
\quad(49.87)^{\mathrm{ab}}\end{array}$ & $8.15^{\text {abcdefg }}$ & $25.04^{\mathrm{defg}}$ & $33.33^{\mathrm{a}}$ & $321.68^{a}$ & $13.47^{\text {abcdefg }}$ & $\begin{array}{l}18.75 \\
(25.63)^{\mathrm{b}}\end{array}$ & $\begin{array}{l}211.33 \\
(14.52)^{\mathrm{a}}\end{array}$ \\
\hline C. bijugum & IG 70018 & $\begin{array}{l}62.50 \\
(52.27)^{\mathrm{abc}}\end{array}$ & $3.61^{\mathrm{a}}$ & $26.77^{\mathrm{ij}}$ & $35.42^{\mathrm{a}}$ & $328.21^{\mathrm{ab}}$ & $15.73^{\mathrm{ij}}$ & $\begin{array}{l}20.83 \\
(27.05)^{\mathrm{bc}}\end{array}$ & $\begin{array}{l}207.33 \\
(14.40)^{\mathrm{a}}\end{array}$ \\
\hline C. bijugum & IG 70022 & $\begin{array}{l}62.50 \\
(52.35)^{\mathrm{abc}}\end{array}$ & $5.17^{\mathrm{abcd}}$ & $26.14^{\text {fghij }}$ & $37.50^{\mathrm{ab}}$ & $358.28^{\text {abcde }}$ & $15.81^{\mathrm{j}}$ & $\begin{array}{l}25.00 \\
(30.00)^{\mathrm{cd}}\end{array}$ & $\begin{array}{l}287.33 \\
(16.95)^{\mathrm{ef}}\end{array}$ \\
\hline $\begin{array}{l}\text { C. reticula- } \\
\text { tum }\end{array}$ & IG 72933 & $\begin{array}{l}58.33 \\
\quad(49.87)^{\mathrm{ab}}\end{array}$ & $9.94^{\text {efgh }}$ & $25.08^{\operatorname{defg}}$ & $41.67^{\mathrm{abc}}$ & $391.62^{\text {cdefg }}$ & $12.95^{\mathrm{abcdef}}$ & $\begin{array}{l}22.92 \\
(28.58)^{\mathrm{bcd}}\end{array}$ & $\begin{array}{l}349.00 \\
(18.68)^{\text {ghi }}\end{array}$ \\
\hline $\begin{array}{l}\text { C. reticula- } \\
\text { tum }\end{array}$ & IG 72953 & $\begin{array}{l}70.83 \\
(57.31)^{\mathrm{abcd}}\end{array}$ & $11.24^{\text {fgh }}$ & $24.57^{\text {cde }}$ & $52.08^{\text {abcde }}$ & $410.63^{\mathrm{fgh}}$ & $13.67^{\text {bcdefgh }}$ & $\begin{array}{l}37.50 \\
(37.73)^{\mathrm{fgh}}\end{array}$ & $\begin{array}{l}344.00 \\
(18.54)^{\mathrm{ghi}}\end{array}$ \\
\hline $\begin{array}{l}\text { C. pinnatifi- } \\
\text { dum }\end{array}$ & PI 510663 & $\begin{array}{l}70.83 \\
(57.37)^{\mathrm{abcd}}\end{array}$ & $9.29^{\text {cdefgh }}$ & $26.74^{\text {hij }}$ & $52.08^{\text {abcde }}$ & $351.44^{\mathrm{abcd}}$ & $15.62^{\mathrm{hij}}$ & $\begin{array}{l}33.33 \\
(35.22)^{\mathrm{fg}}\end{array}$ & $\begin{array}{l}277.00 \\
(16.66)^{\mathrm{def}}\end{array}$ \\
\hline C. judaicum & PI 568217 & $\begin{array}{l}62.50 \\
(52.27)^{\mathrm{abc}}\end{array}$ & $9.58^{\mathrm{defgh}}$ & $26.27^{\text {fghij }}$ & $43.75^{\mathrm{abcd}}$ & $369.78^{\text {bcdef }}$ & $12.19^{\mathrm{abcd}}$ & $\begin{array}{l}31.25 \\
(33.98)^{\mathrm{ef}}\end{array}$ & $\begin{array}{l}223.00 \\
(14.93)^{\mathrm{ab}}\end{array}$ \\
\hline C. bijugum & PI 599046 & $\begin{array}{l}64.58 \\
\quad(53.92)^{\mathrm{abc}}\end{array}$ & $4.74^{\mathrm{abc}}$ & $25.36^{\mathrm{efgh}}$ & $43.75^{\mathrm{abcd}}$ & $341.07^{\mathrm{ab}}$ & $14.23^{\text {efghij }}$ & $\begin{array}{l}22.92 \\
(28.58)^{\mathrm{bcd}}\end{array}$ & $\begin{array}{l}227.00 \\
\quad(15.06)^{\mathrm{abc}}\end{array}$ \\
\hline C. bijugum & PI 599066 & $\begin{array}{l}62.50 \\
\quad(52.27)^{\mathrm{abc}}\end{array}$ & $3.82^{\mathrm{ab}}$ & $25.50^{\text {efghi }}$ & $31.25^{\mathrm{a}}$ & $339.16^{\mathrm{ab}}$ & $14.64^{\text {fghij }}$ & $\begin{array}{l}22.92 \\
\quad(28.58)^{\mathrm{bcd}}\end{array}$ & $\begin{array}{l}216.33 \\
(14.72)^{\mathrm{a}}\end{array}$ \\
\hline C. judaicum & PI 599077 & $\begin{array}{l}87.50 \\
(69.30)^{\mathrm{def}}\end{array}$ & $8.49^{\text {bcdefg }}$ & $24.88^{\text {cdef }}$ & $64.58^{\mathrm{de}}$ & $345.14^{\mathrm{ab}}$ & $15.32^{\text {ghij }}$ & $\begin{array}{l}39.58 \\
\quad(38.98)^{\mathrm{gh}}\end{array}$ & $\begin{array}{l}228.00 \\
(15.11)^{\mathrm{abc}}\end{array}$ \\
\hline $\begin{array}{l}\text { C. pinnatifi- } \\
\text { dum }\end{array}$ & PI 599109 & $\begin{array}{l}75.00 \\
(60.08)^{\text {bcde }}\end{array}$ & $7.24^{\text {abcdef }}$ & $23.63^{\mathrm{abc}}$ & $58.33^{\text {bcde }}$ & $347.50^{\mathrm{abc}}$ & $13.49^{\text {abcdefg }}$ & $\begin{array}{l}35.42 \\
\quad(36.45)^{\mathrm{fg}}\end{array}$ & $\begin{array}{l}272.33 \\
(16.47)^{\text {cdef }}\end{array}$ \\
\hline $\begin{array}{l}\text { C. micro- } \\
\text { phyllum }\end{array}$ & $\begin{array}{l}\text { ICCW } \\
17148\end{array}$ & $\begin{array}{l}79.17 \\
(62.95)^{\text {cdef }}\end{array}$ & $4.32^{\mathrm{ab}}$ & $26.94^{j}$ & $52.08^{\text {abcde }}$ & $340.08^{\mathrm{ab}}$ & $13.56^{\text {abcdefg }}$ & $\begin{array}{l}39.58 \\
(38.98)^{\mathrm{gh}}\end{array}$ & $\begin{array}{l}295.33 \\
(17.19)^{\mathrm{efg}}\end{array}$ \\
\hline C. arietinum & JG $11(\mathrm{C})$ & $\begin{array}{l}89.58 \\
(71.26)^{\mathrm{ef}}\end{array}$ & $17.12^{\mathrm{j}}$ & $22.93^{\mathrm{ab}}$ & $72.92^{\mathrm{e}}$ & $464.73^{\mathrm{i}}$ & $11.63^{\mathrm{a}}$ & $\begin{array}{l}56.25 \\
\quad(48.59)^{\mathrm{i}}\end{array}$ & $\begin{array}{l}382.33 \\
(19.50)^{\mathrm{i}}\end{array}$ \\
\hline C. arietinum & KAK 2 (S) & $\begin{array}{l}91.67 \\
(73.22)^{\mathrm{f}}\end{array}$ & $16.19^{\mathrm{ij}}$ & $22.93^{\mathrm{ab}}$ & $72.92^{\mathrm{e}}$ & $427.42^{\text {ghi }}$ & $12.11^{\mathrm{abc}}$ & $\begin{array}{l}50.00 \\
\quad(45.00)^{\mathrm{i}}\end{array}$ & $\begin{array}{l}351.33 \\
(18.72)^{\mathrm{hi}}\end{array}$ \\
\hline C. arietinum & $\begin{array}{l}\text { ICC } 3137 \\
\text { (S) }\end{array}$ & $\begin{array}{l}87.50 \\
(69.30)^{\mathrm{def}}\end{array}$ & $13.28^{\mathrm{hij}}$ & $22.35^{\mathrm{a}}$ & $70.83^{\mathrm{e}}$ & $446.31^{\mathrm{hi}}$ & $11.77^{\mathrm{ab}}$ & $\begin{array}{l}54.17 \\
\quad(47.40)^{\mathrm{i}}\end{array}$ & $\begin{array}{l}382.00 \\
(19.51)^{\mathrm{i}}\end{array}$ \\
\hline C. arietinum & $\begin{array}{l}\text { ICCL } 86111 \\
\text { (R) }\end{array}$ & $\begin{array}{l}75.00 \\
(60.32)^{\text {bcde }}\end{array}$ & $12.34^{\mathrm{ghi}}$ & $23.88^{\mathrm{bcd}}$ & $62.50^{\text {cde }}$ & $395.94^{\text {defg }}$ & $12.35^{\text {abcde }}$ & $\begin{array}{l}39.58 \\
(38.94)^{\mathrm{gh}}\end{array}$ & $\begin{array}{l}341.00 \\
(18.48)^{\text {ghi }}\end{array}$ \\
\hline \multirow[t]{5}{*}{ C. arietinum } & $\begin{array}{l}\text { ICC 506EB } \\
\text { (R) }\end{array}$ & $\begin{array}{l}81.25 \\
(64.37)^{\text {cdef }}\end{array}$ & $13.38^{\mathrm{hij}}$ & $23.81^{\mathrm{bcd}}$ & $62.50^{\text {cde }}$ & $398.65^{\text {efg }}$ & $12.46^{\text {abcde }}$ & $\begin{array}{l}43.75 \\
\quad(41.41)^{\mathrm{h}}\end{array}$ & $\begin{array}{l}307.00 \\
(17.52)^{\mathrm{fgh}}\end{array}$ \\
\hline & Fp & $<0.001$ & $<0.001$ & $<0.001$ & $<0.001$ & $<0.001$ & $<0.001$ & $<.001$ & $<.001$ \\
\hline & Mean & 58.82 & 8.62 & 24.95 & 50.62 & 371.91 & 13.65 & 34.97 & 16.72 \\
\hline & $\mathrm{SE} \pm$ & 3.69 & 1.39 & 0.42 & 6.27 & 13.59 & 0.58 & 1.20 & 0.47 \\
\hline & $\begin{array}{l}\text { LSD } \\
\qquad(P=0.05)\end{array}$ & 10.92 & 4.10 & 1.23 & 18.54 & 40.22 & 1.71 & 3.54 & 1.35 \\
\hline
\end{tabular}

$C$ commercial cultivar, $S$ susceptible check, $R$ resistant check

The values followed by same alphabet did not differ significantly at $P \leq 0.05$ (DMRT)

${ }^{\#}$ Figures in the parentheses are Angular transformed values; $D A E$ days after initiation of experiment

\#\# Figures in the parentheses are square root $(\sqrt{ } x+0.5)$ transformed values 
significantly among the genotypes grown under glasshouse condition (Table 3). Lowest larval survival on 10th day was observed in larvae reared on artificial diets with the leaf powder of IG 70018 (47.92\%), followed by those reared on diets with ICCW 17148 (52.08\%) and IG 70012 (52.08\%). The highest larval survival was observed on the susceptible check, ICC 3137 (81.25\%). Larval weights were significantly lower when the insects reared on diets with leaf powder of the wild genotypes as compared to that on the susceptible check, KAK $2(14.63 \mathrm{mg})$, except on diets with leaf powder of IG $72933(10.12 \mathrm{mg})$ and IG 599076 (9.15 mg). Lowest larval weight was recorded on ICCW $17148(1.69 \mathrm{mg})$, followed by PI 599046 (2.02 mg). Larval period was longest in insects reared on diets with leaf powder of IG 70018 (27.18 days), followed by ICCW 17148 (26.80 days), while the shorter larval period was observed on JG 11 (24.38 days) followed by ICC 3137 (24.70 days) and KAK 2 (24.80 days).

Pupation was significantly lower in insects reared on diets with leaf powder of ICCW 17148, PI 599046, IG 70018, IG 70012 and IG 70022 (20.83 to $35.42 \%$ ) than those reared on diets with leaf powder of the susceptible check, KAK 2 (50.00\%). Lower pupal weights were observed in insects reared on diets with leaf powder of wild relatives of chickpea ( 313.54 to $362.20 \mathrm{mg}$ ) as compared to those reared on diets with leaf powder of the susceptible check, ICC 3137 (388.23 mg), which was not significantly different from KAK 2 (380.03 mg), ICC 506EB (363.61 mg), JG 11 (362.79 mg) and ICCL 86,111 (362.23 mg).

Pupal period was delayed when the H.armigera larvae were reared on diets with leaf powder of wild relatives of chickpea (ranging from 13.33 days in IG 72953 to 16.14 days in PI 599066) as compared to the larvae reared on diets with leaf powder of the susceptible check, KAK 2 (12.22 days). Lowest adult emergence was observed in insets reared on diets with leaf powder of ICCW 17148 (10.42\%), followed by IG 70018 (14.58\%) and PI 599046 (14.58\%). highest adult emergence was observed in insects reared on KAK 2 (45.83\%). Lowest fecundity of $H$. armigera was observed when the insects were reared on PI 599046 (205.00 eggs/ female), and highest on JG 11 (396.50 eggs/female), which was not significantly different from IG 72953 (342.50 eggs/ female), KAK 2 (329 eggs/female), ICC 3137 (316.50 eggs/ female), ICCW 17148 (316.00 eggs/female) and IG 72933 (303.50 eggs/female).

\section{Biochemical characterization of wild relatives of chickpea}

\section{Protein content}

There were significant differences in protein content among the wild relatives and the cultivated genotypes of chickpea (Table 4). During the post-rainy season 2014-2015, greater amounts of protein content were recorded in KAK $2(16.41 \%)$ and PI 599066 (15.89\%), while lowest was observed in ICC 3137 (11.42\%) which was not significantly different from PI 568217 (11.99\%) and ICCW 17,148 (12.39\%). During the post-rainy season 2015-2016, the C. bijugum genotypes IG 70018, PI 599046, IG 70012, PI 599066, IG 70022 and IG 70006 recorded higher protein content (15.40 to $12.38 \%$ ) than ICC 506EB (8.27\%). Under glasshouse conditions, lowest protein content was recorded in PI 599077 (7.73\%), which was not significantly different from IG 69979 (7.87\%), PI 568217 (8.16\%), PI 599109 $(8.18 \%)$ and ICC 506EB (8.65\%), while highest protein content was recorded in IG 70012 (12.20\%).

\section{Phenol content}

Significant differences were observed in phenol content among the genotypes tested (Table 4). During the post-rainy season 2014-2015, accessions belonging to wild relatives of chickpea exhibited significantly higher amounts of phenols $(6.55 \mathrm{mg} / \mathrm{g}$ in PI 599077 to $7.97 \mathrm{mg} / \mathrm{g}$ in PI 599046) as compared to the cultivated chickpea ( $5.93 \mathrm{mg} / \mathrm{g}$ in ICCL 86111 to $6.15 \mathrm{mg} / \mathrm{g}$ in ICC $506 \mathrm{~EB})$, except in C. reticulatum, IG $72953(4.10 \mathrm{mg} / \mathrm{g})$ and IG $72933(4.52 \mathrm{mg} / \mathrm{g})$ and $C$. chrossanicum, IG $599076(6.15 \mathrm{mg} / \mathrm{g})$. During the post-rainy season 2015-2016, highest phenol content was observed in PI $599046(6.50 \mathrm{mg} / \mathrm{g})$, which was not significantly different from IG $70006(6.41 \mathrm{mg} / \mathrm{g})$, while lowest protein content was recorded in IG $599076(4.07 \mathrm{mg} / \mathrm{g})$ which was not significantly different from the susceptible check, ICC 3137 $(4.16 \mathrm{mg} / \mathrm{g})$. Under glasshouse conditions, phenol content varied significantly among genotypes tested, and ranged from $3.06 \mathrm{mg} / \mathrm{g}$ (ICC 3137) to $5.90 \mathrm{mg} / \mathrm{g}$ (IG 70006).

\section{Total soluble sugars}

Significant differences were observed in total soluble sugars among different genotypes of chickpea (Table 4). During the post-rainy season 2014-2015, the genotypes ICCW 17148, PI 568217, IG 599076, PI 599109, IG 72933, PI 510663, IG 72953, IG 69,979 and PI 599046 had significantly lower amounts of total soluble sugars $(8.04$ to $9.61 \%)$ than the susceptible check, KAK 2 (13.60\%). During the post-rainy season 2015-2016, lower amounts of total soluble sugars were recorded in IG 599076 (10.35\%), followed by IG 69979 $(11.05 \%)$, while highest amounts of soluble sugars were recorded in KAK 2 (17.18\%), which was not significantly different from ICC 506EB (16.54\%), ICC 3137 (16.05\%) and JG 11 (15.86\%). Under glasshouse conditions, amounts of total soluble sugars were significantly lower in IG 72933 (7.38\%), PI $599077(7.87 \%)$ and IG $599076(7.91 \%)$ than in IG $70022(16.35 \%)$ and IG 70018 (14.96\%). 
Table 3 Expression of resistance to H. armigera in wild relatives of chickpea grown under glasshouse conditions (diet incorporation assay)

\begin{tabular}{|c|c|c|c|c|c|c|c|c|c|}
\hline Species & Genotype & $\begin{array}{l}\text { Larval } \\
\text { survival } 10 \\
\text { DAE }(\%)\end{array}$ & $\begin{array}{l}\text { Mean larval } \\
\text { weight } 10 \\
\text { DAE (mg) }\end{array}$ & $\begin{array}{l}\text { Larval } \\
\text { period } \\
\text { (days) }\end{array}$ & $\begin{array}{l}\text { Pupation } \\
(\%)\end{array}$ & $\begin{array}{l}\text { Mean pupal } \\
\text { weight (mg) }\end{array}$ & $\begin{array}{l}\text { Pupal } \\
\text { period } \\
\text { (days) }\end{array}$ & $\begin{array}{l}\text { Adult emer- } \\
\text { gence }(\%)\end{array}$ & $\begin{array}{l}\text { Fecundity } \\
\text { (eggs/ } \\
\text { female) }\end{array}$ \\
\hline $\begin{array}{l}\text { C. chros- } \\
\text { sanicum }\end{array}$ & IG 599076 & $60.42^{\mathrm{abcd}}$ & $9.15^{\text {bcdef }}$ & $25.83^{\mathrm{abcdef}}$ & $41.67^{\mathrm{cd}}$ & $362.20^{\text {cde }}$ & $14.78^{\text {bcdef }}$ & $\begin{array}{l}35.42 \\
(36.51)^{\text {fghi }}\end{array}$ & $\begin{array}{l}282.50 \\
(16.82)^{\mathrm{abc}}\end{array}$ \\
\hline C. cuneatum & IG 69979 & $60.42^{\text {abcd }}$ & $8.65^{\text {abcdef }}$ & $25.41^{\text {abcde }}$ & $37.50^{\mathrm{bcd}}$ & $352.88^{\mathrm{bcd}}$ & $13.89^{\text {abcdef }}$ & $\begin{array}{l}29.17 \\
(32.46)^{\text {cdef }}\end{array}$ & $\begin{array}{l}236.50 \\
(15.34)^{a b}\end{array}$ \\
\hline C. bijugum & IG 70006 & $58.33^{\mathrm{abc}}$ & $4.97^{\mathrm{abc}}$ & $26.50^{\mathrm{def}}$ & $43.75^{\mathrm{cd}}$ & $356.19^{\text {bcde }}$ & $14.73^{\text {abcdef }}$ & $\begin{array}{l}25.00 \\
(29.92)^{\mathrm{bcd}}\end{array}$ & $\begin{array}{l}249.00 \\
\quad(15.79)^{a b}\end{array}$ \\
\hline C. bijugum & IG 70012 & $52.08^{\mathrm{ab}}$ & $6.52^{\mathrm{abcd}}$ & $26.37^{\text {cdef }}$ & $35.42^{\mathrm{abcd}}$ & $351.79^{\mathrm{bcd}}$ & $15.50^{\mathrm{def}}$ & $\begin{array}{l}22.92 \\
(28.58)^{\mathrm{bc}}\end{array}$ & $\begin{array}{l}241.50 \\
\quad(15.51)^{a b}\end{array}$ \\
\hline C. bijugum & IG 70018 & $47.92^{\mathrm{a}}$ & $3.42^{\mathrm{ab}}$ & $27.18^{\mathrm{f}}$ & $33.33^{\mathrm{abc}}$ & $328.98^{\mathrm{ab}}$ & $15.90^{\mathrm{ef}}$ & $\begin{array}{l}14.58 \\
(22.40)^{\mathrm{a}}\end{array}$ & $\begin{array}{l}237.50 \\
\quad(15.38)^{a b}\end{array}$ \\
\hline C. bijugum & IG 70022 & $58.33^{\mathrm{abc}}$ & $3.94^{\mathrm{ab}}$ & $26.61^{\mathrm{def}}$ & $35.42^{\mathrm{abcd}}$ & $343.77^{\mathrm{bc}}$ & $15.00^{\text {bcdef }}$ & $\begin{array}{l}22.92 \\
(28.39)^{\mathrm{bc}}\end{array}$ & $\begin{array}{l}263.00 \\
\quad(16.16)^{a b}\end{array}$ \\
\hline $\begin{array}{l}\text { C. reticula- } \\
\text { tum }\end{array}$ & IG 72933 & $70.83^{\text {bcde }}$ & $10.12^{\text {bcdef }}$ & $25.67^{\mathrm{abcdef}}$ & $41.67^{\mathrm{cd}}$ & $354.20^{\mathrm{bcd}}$ & $13.57^{\text {abcde }}$ & $\begin{array}{l}31.25 \\
(33.98)^{\operatorname{defg}}\end{array}$ & $\begin{array}{l}303.50 \\
(17.38) \mathrm{abc}\end{array}$ \\
\hline $\begin{array}{l}\text { C. reticula- } \\
\text { tum }\end{array}$ & IG 72953 & $68.75^{\text {bcde }}$ & $7.61^{\text {abcde }}$ & $25.96^{\mathrm{abcdef}}$ & $41.67^{\mathrm{cd}}$ & $353.83^{\text {bcd }}$ & $13.33^{\mathrm{abcd}}$ & $\begin{array}{l}29.17 \\
(32.63)^{\text {cdef }}\end{array}$ & $\begin{array}{l}342.50 \\
(18.51)^{b c}\end{array}$ \\
\hline $\begin{array}{l}\text { C. pinnatifi- } \\
\text { dum }\end{array}$ & PI 510663 & $64.58^{\text {abcde }}$ & $6.91^{\mathrm{abcd}}$ & $25.50^{\text {abcde }}$ & $41.67^{\mathrm{cd}}$ & $357.12^{\text {bcde }}$ & $15.07^{\text {cdef }}$ & $\begin{array}{l}33.33 \\
(35.26)^{\text {efghi }}\end{array}$ & $\begin{array}{l}294.50 \\
(17.15)^{a b c}\end{array}$ \\
\hline C. judaicum & PI 568217 & $66.67^{\text {abcde }}$ & $8.23^{\text {abcdef }}$ & $26.00^{\mathrm{abcdef}}$ & $47.92^{\mathrm{cd}}$ & $357.68^{\text {bcde }}$ & $14.50^{\mathrm{abcdef}}$ & $\begin{array}{l}37.50 \\
(37.73)^{\mathrm{ghi}}\end{array}$ & $\begin{array}{l}208.00 \\
\quad(14.43)^{\mathrm{a}}\end{array}$ \\
\hline C. bijugum & PI 599046 & $58.33^{\mathrm{abc}}$ & $2.02^{\mathrm{a}}$ & $26.10^{\text {bcdef }}$ & $22.92^{\mathrm{ab}}$ & $342.45^{\mathrm{abc}}$ & $14.21^{\mathrm{abcdef}}$ & $\begin{array}{l}14.58 \\
(22.40)^{\mathrm{a}}\end{array}$ & $\begin{array}{l}205.00 \\
(14.32)^{\mathrm{a}}\end{array}$ \\
\hline C. bijugum & PI 599066 & $60.42^{\text {abcd }}$ & $6.07^{\mathrm{abcd}}$ & $25.84^{\text {abcdef }}$ & $43.75^{\mathrm{cd}}$ & $349.91^{\text {bcd }}$ & $16.14^{\mathrm{f}}$ & $\begin{array}{l}27.08 \\
(31.21)^{\text {bcde }}\end{array}$ & $\begin{array}{l}212.50 \\
(14.59)^{\mathrm{a}}\end{array}$ \\
\hline C. judaicum & PI 599077 & $60.42^{\text {abcd }}$ & $7.07^{\mathrm{abcd}}$ & $25.80^{\mathrm{abcdef}}$ & $45.83^{\mathrm{cd}}$ & $359.62^{\text {bcde }}$ & $13.86^{\text {abcdef }}$ & $\begin{array}{l}31.25 \\
(33.98)^{\operatorname{defgh}}\end{array}$ & $\begin{array}{l}252.50 \\
(15.90)^{a b}\end{array}$ \\
\hline $\begin{array}{l}\text { C. pinnatifi- } \\
\text { dum }\end{array}$ & PI 599109 & $62.50^{\text {abcde }}$ & $5.74^{\mathrm{abcd}}$ & $26.37^{\text {cdef }}$ & $39.58^{\mathrm{cd}}$ & $359.20^{\text {bcde }}$ & $14.60^{\mathrm{abcdef}}$ & $\begin{array}{l}20.83 \\
(27.05)^{\mathrm{b}}\end{array}$ & $\begin{array}{l}241.50 \\
(15.50)^{a b}\end{array}$ \\
\hline $\begin{array}{l}\text { C. micro- } \\
\text { phyllum }\end{array}$ & $\begin{array}{l}\text { ICCW } \\
17148\end{array}$ & $52.08^{\mathrm{ab}}$ & $1.69^{\mathrm{a}}$ & $26.80^{\mathrm{ef}}$ & $20.83^{\mathrm{a}}$ & $313.54^{\mathrm{a}}$ & $15.85^{\mathrm{def}}$ & $\begin{array}{l}10.42 \\
(18.74)^{\mathrm{a}}\end{array}$ & $\begin{array}{l}316.00 \\
(17.78) \mathrm{bc}\end{array}$ \\
\hline C. arietinum & JG $11(\mathrm{C})$ & $79.17^{\mathrm{de}}$ & $11.16^{\text {cdef }}$ & $24.38^{\mathrm{a}}$ & $47.92^{\mathrm{cd}}$ & $362.79^{\text {cde }}$ & $12.92^{\mathrm{abc}}$ & $\begin{array}{l}39.58 \\
(38.94)^{\mathrm{gij}}\end{array}$ & $\begin{array}{l}396.50 \\
(19.91)^{\mathrm{c}}\end{array}$ \\
\hline C. arietinum & KAK 2 (S) & $75.00^{\text {cde }}$ & $14.63^{\mathrm{f}}$ & $24.80^{\mathrm{abc}}$ & $50.00^{\mathrm{d}}$ & $380.03^{\mathrm{de}}$ & $12.22^{\mathrm{a}}$ & $\begin{array}{l}45.83 \\
\quad(42.60)^{\mathrm{j}}\end{array}$ & $\begin{array}{l}329.00 \\
\quad(18.14)^{\mathrm{ab}}\end{array}$ \\
\hline C. arietinum & $\begin{array}{l}\text { ICC } 3137 \\
\text { (S) }\end{array}$ & $81.25^{\mathrm{e}}$ & $14.34^{\mathrm{ef}}$ & $24.70^{\mathrm{ab}}$ & $47.92^{\mathrm{cd}}$ & $388.23^{\mathrm{e}}$ & $12.50^{\mathrm{ab}}$ & $\begin{array}{l}37.50 \\
(37.65)^{\text {ghi }}\end{array}$ & $\begin{array}{l}316.50 \\
(17.80)^{\mathrm{bc}}\end{array}$ \\
\hline C. arietinum & $\begin{array}{l}\text { ICCL } 86111 \\
\text { (R) }\end{array}$ & $70.83^{\text {bcde }}$ & $11.29^{\text {cdef }}$ & $25.39^{\text {abcde }}$ & $45.83^{\mathrm{cd}}$ & $362.23^{\text {cde }}$ & $14.35^{\text {abcdef }}$ & $\begin{array}{l}33.33 \\
(35.26)^{\mathrm{efghi}}\end{array}$ & $\begin{array}{l}272.50 \\
\quad(16.50)^{\mathrm{ab}}\end{array}$ \\
\hline \multirow[t]{5}{*}{ C. arietinum } & $\begin{array}{l}\text { ICC 506EB } \\
\text { (R) }\end{array}$ & $68.75^{\text {bcde }}$ & $12.35^{\mathrm{def}}$ & $25.02^{\mathrm{abcd}}$ & $45.83^{\mathrm{cd}}$ & $363.61^{\text {cde }}$ & $13.66^{\mathrm{abcdef}}$ & $\begin{array}{l}33.33 \\
(35.22)^{\text {efghi }}\end{array}$ & $\begin{array}{l}275.00 \\
(16.56)^{\mathrm{ab}}\end{array}$ \\
\hline & $\mathrm{Fp}$ & 0.039 & $<0.001$ & 0.035 & 0.01 & 0.015 & 0.04 & $<.001$ & 0.02 \\
\hline & Mean & 63.85 & 7.79 & 25.81 & 40.52 & 355.01 & 14.33 & 32.05 & 16.47 \\
\hline & $\mathrm{SE} \pm$ & 5.83 & 2.09 & 0.48 & 4.66 & 9.43 & 0.73 & 1.47 & 0.92 \\
\hline & $\begin{array}{l}\text { LSD } \\
\qquad(P=0.05)\end{array}$ & 17.27 & 5.86 & 1.41 & 13.79 & 27.92 & 2.16 & 4.34 & 2.73 \\
\hline
\end{tabular}

$C$ commercial cultivar, $S$ susceptible check, $R$ resistant check

The values followed by same alphabet did not differ significantly at $P \leq 0.05$ (DMRT)

${ }^{\#}$ Figures in the parentheses are Angular transformed values; $D A E$ days after initiation of experiment

${ }^{\# \#}$ Figures in the parentheses are square root $(\sqrt{ } x+0.5)$ transformed values 


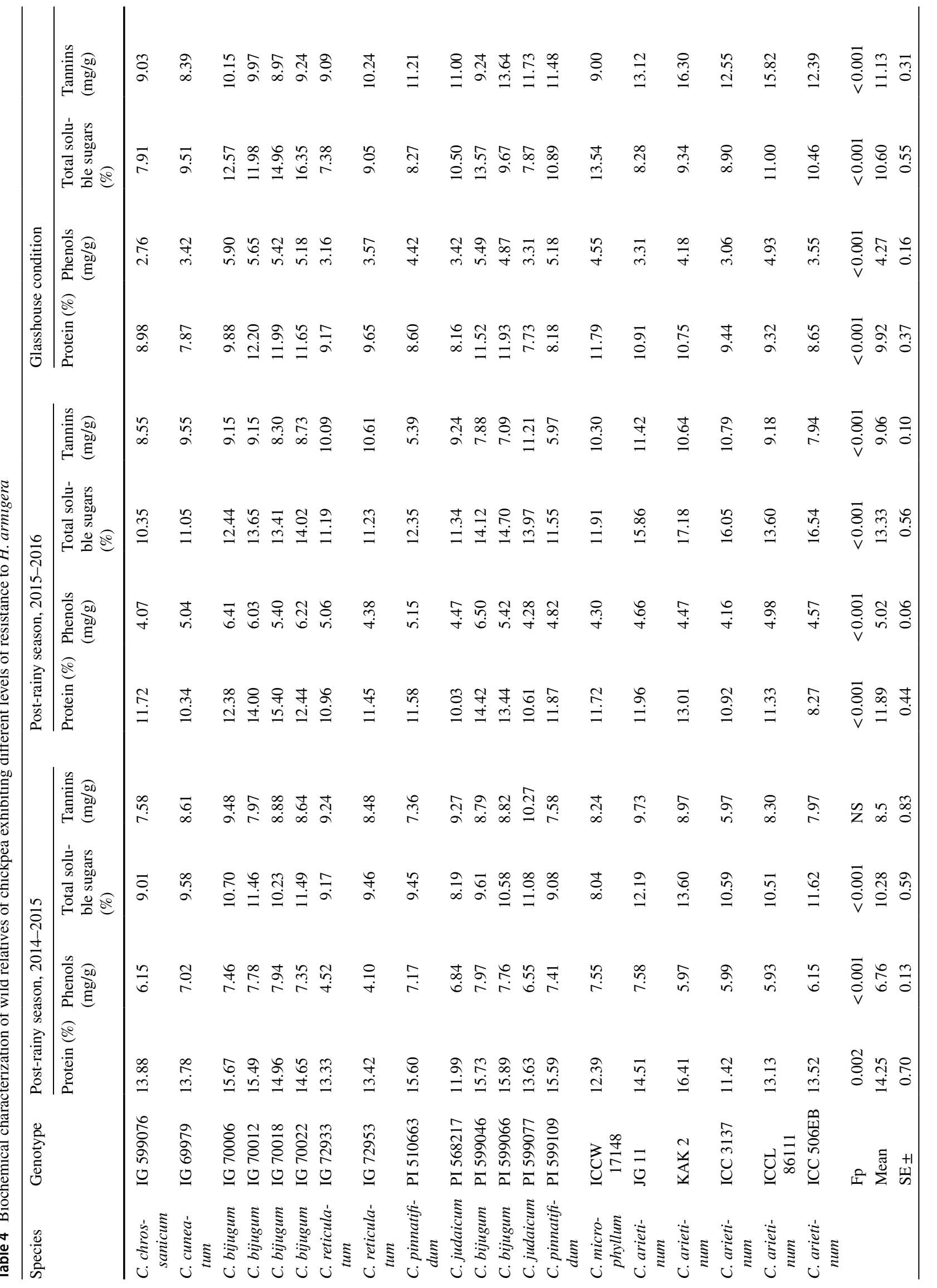




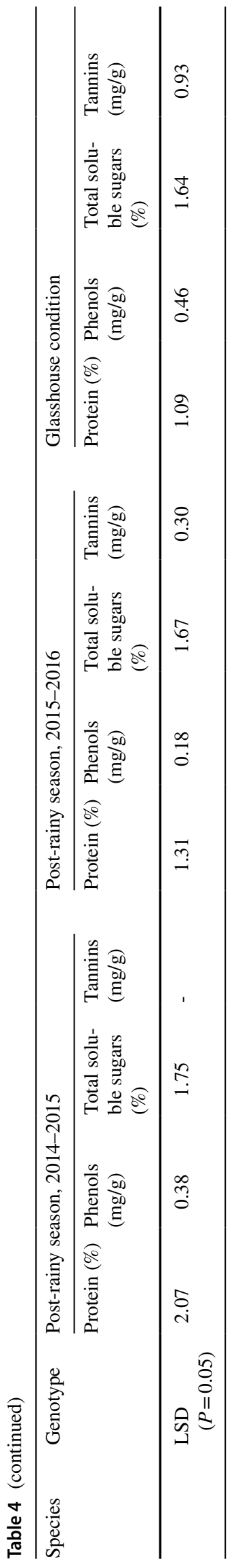

Tannin content

During the post-rainy season 2014-2015, there were no significant differences in tannin content among the genotypes tested (Table 4). During the post-rainy season 2015-2016, tannin content was lower in PI $510663(5.39 \mathrm{mg} / \mathrm{g})$ and PI $599109(5.97 \mathrm{mg} / \mathrm{g})$ as compared to JG $11(11.42 \mathrm{mg} / \mathrm{g})$ and PI $599077(11.21 \mathrm{mg} / \mathrm{g})$. Significant differences were observed in tannin content among different chickpea genotypes under glasshouse conditions. KAK2 recorded higher tannin content $(16.30 \mathrm{mg} / \mathrm{g})$ than in IG $69979(8.39 \mathrm{mg} / \mathrm{g})$.

\section{Flavonoids content in wild relatives of chickpea estimated through HPLC fingerprints}

Significant differences were observed in flavonoid compounds identified through HPLC fingerprints at different retention times (RT from 8.11 to $25.70 \mathrm{~min}$ ) among the genotypes tested (Table 5). Among the flavonoid compounds, chlorogenic acid (RT $8.11 \mathrm{~min}$ ) was present only in four genotypes, IG $70012(2.90 \mathrm{mg} / \mathrm{g})$, PI 599066 (2.43 mg/g), KAK $2(1.42 \mathrm{mg} / \mathrm{g})$ and ICC $3137(0.36 \mathrm{mg} / \mathrm{g})$, while ferulic acid (RT $12.68 \mathrm{~min}$ ), naringin (RT $13.01 \mathrm{~min}$ ), 3,4-dihydroxy flavones (RT $17.43 \mathrm{~min}$ ) and naringenin (RT $19.78 \mathrm{~min}$ ) were present in a few genotypes of the wild relatives of chickpea, but were absent in the cultivated chickpea. Quercetin (RT $17.79 \mathrm{~min}$ ) was present in all the genotypes, except in ICC $506 \mathrm{~EB}$, and the highest concentration was recorded in PI $599046(12.31 \mathrm{mg} / \mathrm{g})$, which was not significantly different from IG $70022(12.16 \mathrm{mg} / \mathrm{g})$. Lowest was amount of quercetin were recorded in ICC $3137(0.55 \mathrm{mg} / \mathrm{g})$, which was on par with ICCL $86111(0.57 \mathrm{mg} / \mathrm{g})$, JG $11(0.67 \mathrm{mg} / \mathrm{g})$ and IG $69979(0.74 \mathrm{mg} / \mathrm{g})$. Genistein (RT $20.39 \mathrm{~min}$ ) was present in all the genotypes, and its highest concentration was recorded in IG $70022(9.57 \mathrm{mg} / \mathrm{g})$, followed by PI 599066 $(9.07 \mathrm{mg} / \mathrm{g})$, while lowest amounts were recorded in ICC 506EB $(0.42 \mathrm{mg} / \mathrm{g})$. Formononetin (RT $22.76 \mathrm{~min}$ ) was present in all the genotypes, except in IG 69979, while highest concentration was present in IG $70018(4.39 \mathrm{mg} / \mathrm{g})$ and PI $599046(4.36 \mathrm{mg} / \mathrm{g})$ than in (ICCW 17148 and ICC 3137 $0.60 \mathrm{mg} / \mathrm{g}$ ). Biochanin-A (RT $25.70 \mathrm{~min}$ ) was present in all the genotypes, except in IG 69979 and IG 70,018. Greater amounts of Biochanin-A were recorded in IG 599076 $(8.68 \mathrm{mg} / \mathrm{g})$ and PI $568217(6.23 \mathrm{mg} / \mathrm{g})$ than in PI 599109 $(0.89 \mathrm{mg} / \mathrm{g})$ and PI $599066(0.93 \mathrm{mg} / \mathrm{g})$.

\section{Association of biochemical components in wild relatives of chickpea with survival and development of $\mathrm{H}$. armigera}

Biochemical components of wild relatives showed significant influence on survival and development of $H$. armigera (Table 6). Proteins content was significantly and negatively 


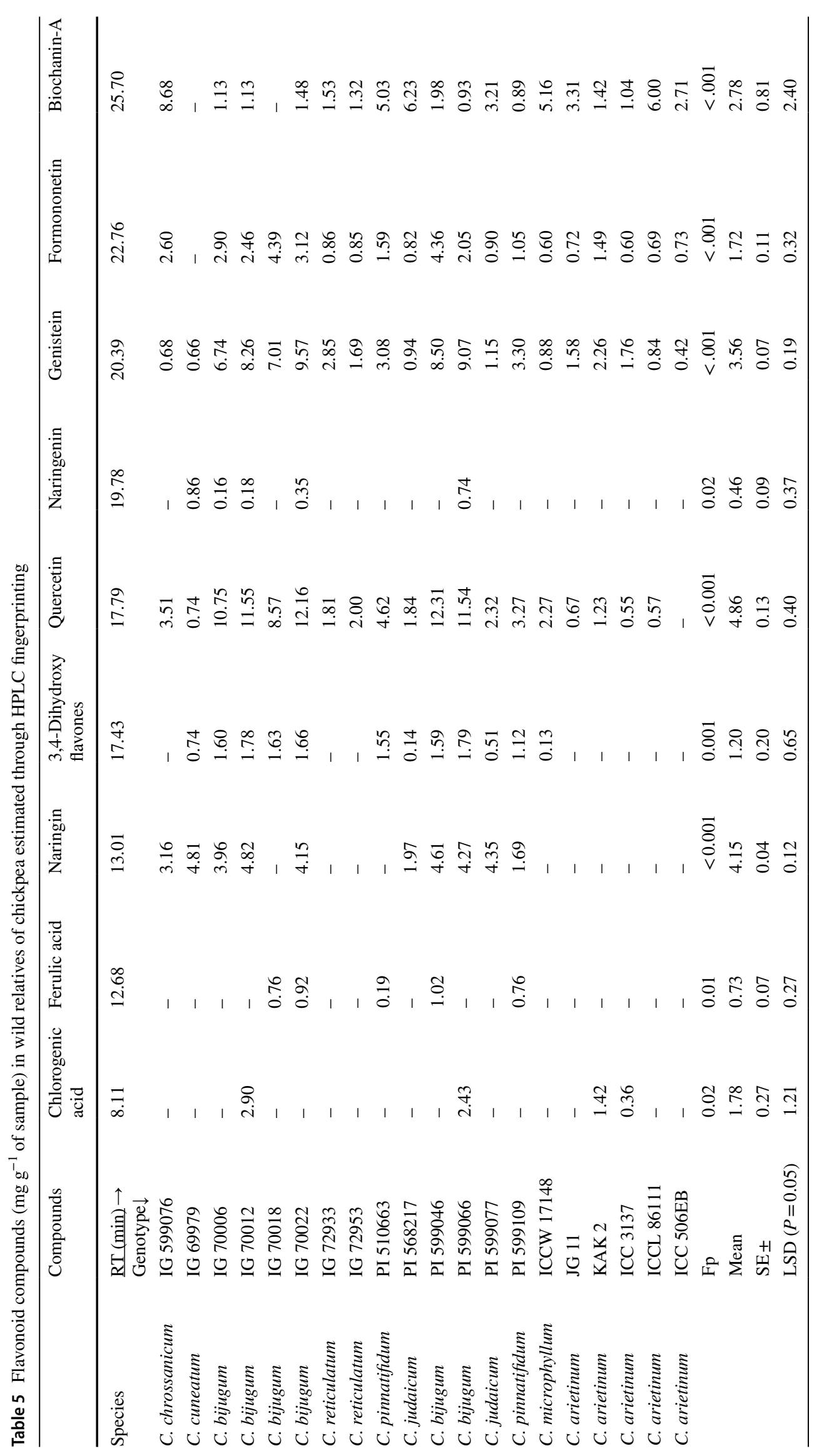


correlated with larval weight, pupation and adult emergence ( $r=-0.26,-0.31$ and -0.26 , respectively). Phenols content was significantly and negatively correlated with larval weight, pupation, pupal weight, adult emergence and fecundity $(r=-0.35,-0.41,-0.25,-0.37$ and -0.30 , respectively), while pupal period was correlated $(r=0.27)$ positively. Total soluble sugars were positively correlated with pupation $(r=0.35)$ and pupal weight $(r=0.25)$, but negatively correlated with larval period $(r=-0.21)$. Tannins showed a significant and positive association with larval weight, pupation and adult emergence $(r=0.28,0.25$ and 0.25 , respectively).

\section{Association of flavonoids with survival and development of $\boldsymbol{H}$. armigera}

Significant correlations were observed between flavonoid content of wild relatives of chickpea and the biological parameters of $H$. armigera (Table 7). Chlorogenic acid showed a significant and negative correlation with larval survival, larval weight, pupation, pupal weight, adult emergence and fecundity $(r=-0.90,-0.80,-0.84,-0$. $90,-0.84$ and -0.82 , respectively), while larval period $(r=0.93)$ and pupal period $(r=0.69)$ were correlated positively. Amounts of ferulic acid were significantly and negatively correlated with pupation, pupal period, adult emergence and fecundity $(r=-0.64,-0.46,-0.70$ and -0.51 , respectively). Naringin showed a significant and negative correlation with larval weight $(r=-0.50)$, pupal weight $(r=-0.44)$ and fecundity $(r=-0.71) .3,4$-Dihydroxy flavones had significant negative correlation with larval survival $(r=-0.58)$, pupation $(r=-0.45)$ and adult emergence $(r=-0.53)$. Quercetin was significantly and negatively correlated with all the biological parameters, except larval period $(r=0.50)$ and pupal period $(r=0.67)$. Naringenin was significantly and negatively correlated with larval weight $(r=-0.62)$ and fecundity $(r=-0.62)$, but positively correlated with adult emergence $(r=0.80)$. Genistein showed a significant and positive correlation with pupal period $(r=0.58)$ and negative correlation with pupation, pupal weight, adult emergence and fecundity $(r=-0.58,-0.47,-0.69$ and -0.48 , respectively). Formononetin showed a significant and positive correlation with larval and pupal periods ( $r=0.53$ and 0.63 , respectively), but a negative correlation was observed with the other biological parameters. Among the flavonoid

Table 6 Association of biochemical components in wild relatives of chickpea with survival and development of $H$. armigera

\begin{tabular}{|c|c|c|c|c|c|c|c|c|}
\hline & $\begin{array}{l}\text { Larval } \\
\text { survival } \\
(\%)\end{array}$ & Larval weight (mg) & $\begin{array}{l}\text { Larval } \\
\text { period } \\
\text { (days) }\end{array}$ & Pupation (\%) & Pupal weight (mg) & $\begin{array}{l}\text { Pupal } \\
\text { period } \\
\text { (days) }\end{array}$ & $\begin{array}{l}\text { Adult emergence } \\
(\%)\end{array}$ & Fecundity \\
\hline Protein $(\%)$ & 0.05 & $-0.26^{*}$ & 0.01 & $-0.31 *$ & -0.14 & 0.16 & $-0.26^{*}$ & -0.11 \\
\hline Phenols (mg/g) & -0.03 & $-0.35^{* *}$ & 0.13 & $-0.41 * *$ & $-0.25^{*}$ & $0.27 *$ & $-0.37 * *$ & $-0.30 *$ \\
\hline $\begin{array}{c}\text { Total soluble } \\
\text { sugars (\%) }\end{array}$ & -0.03 & 0.15 & $-0.21 *$ & $0.35^{* *}$ & $0.25 *$ & -0.08 & 0.11 & 0.06 \\
\hline Tannins (mg/g) & 0.07 & $0.28 *$ & -0.06 & $0.25^{*}$ & 0.19 & -0.15 & $0.25 *$ & 0.17 \\
\hline
\end{tabular}

*,** Correlation coefficients significant at $P \leq 0.05$ and 0.01 , respectively

Table 7 Association of flavonoids in wild relatives of chickpea with survival and development of $H$. armigera

\begin{tabular}{|c|c|c|c|c|c|c|c|c|}
\hline & $\begin{array}{l}\text { Larval survival } \\
(\%)\end{array}$ & $\begin{array}{l}\text { Larval weight } \\
(\mathrm{mg})\end{array}$ & $\begin{array}{l}\text { Larval } \\
\text { period } \\
\text { (days) }\end{array}$ & Pupation (\%) & $\begin{array}{l}\text { Pupal weight } \\
\text { (mg) }\end{array}$ & $\begin{array}{l}\text { Pupal period } \\
\text { (days) }\end{array}$ & $\begin{array}{l}\text { Adult emer- } \\
\text { gence }(\%)\end{array}$ & Fecundity \\
\hline $\begin{array}{l}\text { Chlorogenic } \\
\text { acid }\end{array}$ & $-0.90 * *$ & $-0.80 * *$ & $0.93 * *$ & $-0.84 * *$ & $-0.90 * *$ & $0.69 * *$ & $-0.84 * *$ & $-0.82 * *$ \\
\hline Ferulic acid & -0.29 & 0.36 & -0.13 & $-0.64 * *$ & -0.17 & $-0.46^{*}$ & $-0.70 * *$ & $-0.51^{*}$ \\
\hline Naringin & 0.25 & $-0.50 *$ & -0.31 & -0.13 & $-0.44 *$ & -0.13 & -0.16 & $-0.71 * *$ \\
\hline $\begin{array}{l}\text { 3,4-Dihydroxy } \\
\text { flavone }\end{array}$ & $-0.58 * *$ & 0.11 & -0.12 & $-0.45^{*}$ & -0.19 & 0.24 & $-0.53^{*}$ & 0.06 \\
\hline Quercetin & $-0.52 *$ & $-0.51 *$ & $0.50 *$ & $-0.68 * *$ & $-0.60 * *$ & $0.67 * *$ & $-0.77 * *$ & $-0.59 * *$ \\
\hline Naringenin & 0.05 & $-0.62 * *$ & -0.39 & 0.19 & 0.24 & -0.18 & $0.80 * *$ & $-0.62 * *$ \\
\hline Genistein & -0.30 & -0.37 & 0.40 & $-0.58 * *$ & $-0.47 *$ & $0.58 * *$ & $-0.69 * *$ & $-0.48^{*}$ \\
\hline Formononetin & -0.41 & $-0.49 *$ & $0.53^{*}$ & $-0.71 * *$ & $-0.57 * *$ & $0.60 * *$ & $-0.73 * *$ & $-0.59 * *$ \\
\hline Biochanin A & -0.23 & -0.17 & 0.20 & -0.13 & -0.15 & 0.05 & 0.05 & -0.06 \\
\hline
\end{tabular}

*,** Correlation coefficients significant at $P \leq 0.05$ and 0.01 , respectively 
compounds, Biochanin-A did not show any significant correlations with the biological parameters of $H$. armigera.

\section{Similarity matrix analysis}

Similarity matrix analysis based on biochemical characters of wild relatives of chickpea and survival and development of $H$. armigera on artificial diet with lyophilized leaf powders of different genotypes of chickpea and its wild relatives separated the test genotypes into seven groups (coefficient 0.95) (Fig. 1). The genotypes belonging to C. chrossanicum (IG 599076), C. cuneatum (IG 69979), C. judaicum (PI 568217 and PI 599077) and C. pinnatifidum (PI 599109 and PI 510663) were grouped together. The genotypes belonging to $C$. reticulatum, which is the progenitor of cultivated chickpea (IG 72933 and IG 72953) were placed in one group, and were closer to the other group consisting of cultivated resistant checks, ICCL 86111 and ICC 506EB. All the genotypes belonging to C. bijugum (IG 70006, IG 70012, PI 599066, PI 599046, IG 70022 and IG 70018) were placed in one group. The genotype, ICCW 17148 which belongs to the tertiary gene pool (C. microphyllum) was placed independently, while the susceptible check, KAK 2 was grouped along with the commercial check, JG 11, which was nearer to another susceptible check, ICC 3137.

\section{Discussion}

Significant differences were observed in survival and development of $H$. armigera on artificial diet impregnated with lyophilized leaf powders of different genotypes of wild relatives of chickpea across the seasons. The wild relatives exhibited high levels of antibiosis to $H$. armigera as compared to cultivated chickpea in terms of lower larval survival, pupation and adult emergence, decreased larval and pupal weights, prolonged larval and pupal developmental periods and reduced fecundity. The genotypes, IG 70018, IG 70012, PI 599066, PI 599046, IG 70006 and IG 70022 (C. bijugum) exhibited high levels of resistance, while IG 69979 (C. cuneatum), ICCW 17148 (C. microphyllum), PI 599077, PI 568217 (C. judaicum) and IG 599076 (C. chrossanicum) exhibited moderate levels of resistance in terms of reduced survival, lower weights, delayed developmental periods
Fig. 1 Dendrogram representing similarities between different accessions of wild relatives of chickpea based on biochemical characters and expression of resistance to $H$. armigera

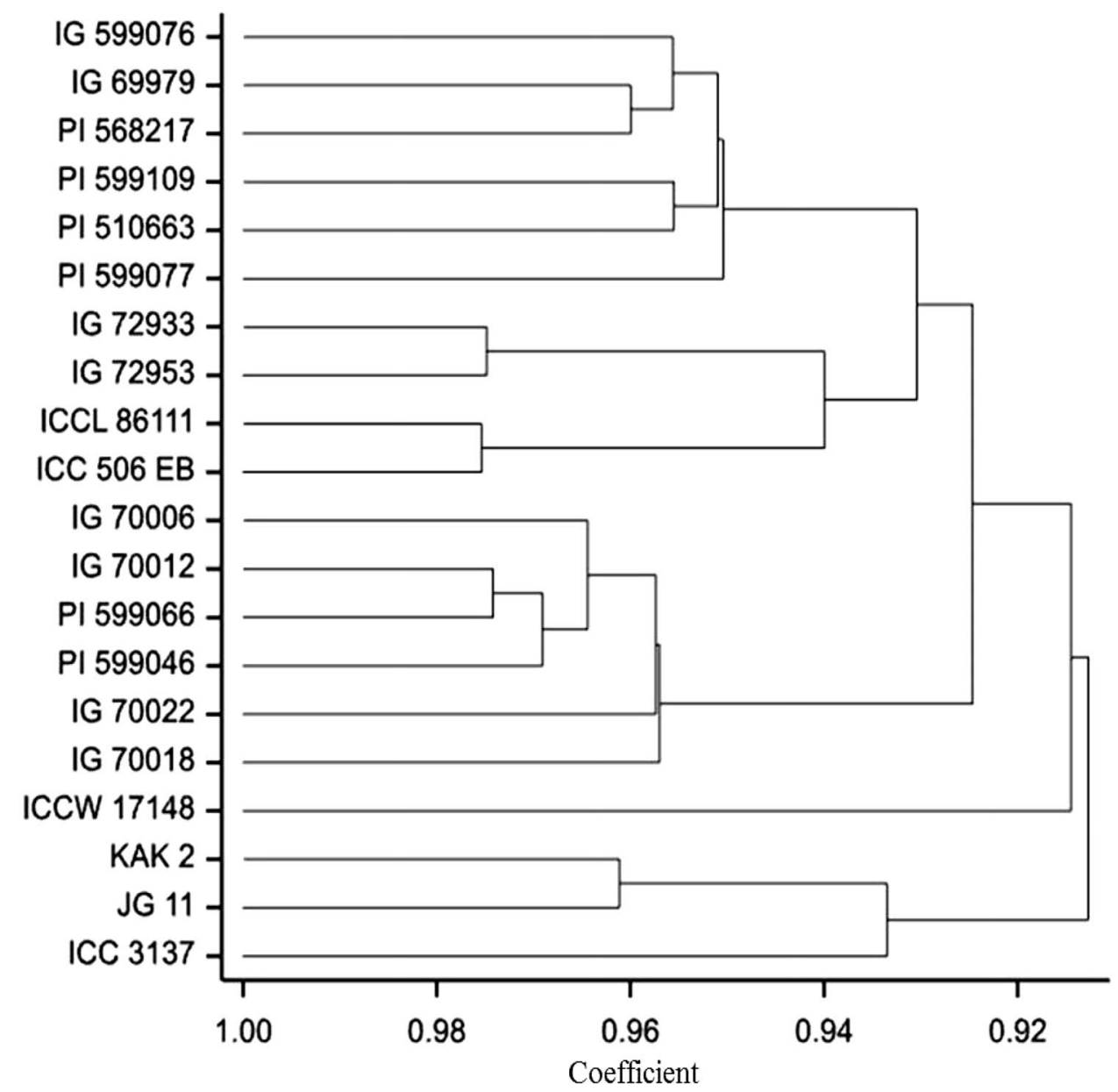


and reduced fecundity of $H$. armigera as compared to the susceptible checks, KAK 2 and ICC 3137 . Higher levels of antibiosis against $H$. armigera in wild relatives as compared to cultigen in terms of reduced survival and delayed developmental periods had also been reported in earlier studies in chickpea (Sharma et al. 2005a, 2006) and pigeonpea (Sujana et al. 2008; Shanower et al. 1997). Narayanamma et al. (2008) also reported that, $F_{1}$ hybrids based on resistant genotypes of chickpea recorded lower larval survival, pupation, and pupal weights as compared to the susceptible check, suggesting the transfer of antibiosis mechanism of resistance to the progeny from resistant parents. The studies indicated that antibiosis seems to be the major component of resistance to $H$. armigera in the wild relatives of chickpea, which might be due to higher amounts of plant secondary metabolites or poor nutritional quality.

Significant differences were observed in biochemical composition among different accessions of wild relatives of chickpea. Proteins and phenols showed negative correlation with larval weight, pupation and adult emergence. Phenols also showed a negative correlation with pupal weight, fecundity, but positive correlation with pupal period. However, Kanchana et al. (2005) reported that protein content had positive correlation with pod damage in cultivated chickpea. These differences could be due to presence of higher amounts of protease inhibitors in wild relatives as compared to the cultivated genotypes (Patankar et al. 1999; Parde et al. 2012; Udamale et al. 2013). Protease inhibitors are reserve proteins present in plants that inhibit insect feeding and digestion of the ingested food by the insects (Blanco-Labra et al. 1995). Chickpea protease inhibitors exhibit differential inhibitory activity against $\mathrm{H}$. armigera gut proteinases (Giri et al. 1998). The antibiosis effects of protease inhibitors such as extended larval period, reduction in larval weight, survival and adult emergence was observed in $H$. armigera fed on diet with chickpea trypsin inhibitor (Kansal et al. 2008). Higher phenolic content in resistant genotypes as compared to the susceptible ones might contribute to resistance against H. armigera (Rupalighodeswar et al. 2003; Kaur et al. 2014). The phenols accumulated in host plant lead to toxicity in insects (Walling 2000; Bhonwong et al. 2009) by increasing the defensive enzyme activity and mediating the transduction pathways, which results in oxidation of toxic substances such as quinines (Maffei et al. 2007; Bhonwong et al. 2009). Several other authors have also reported that total phenols exhibited significant negative correlation with percent pod damage by $H$. armigera (Girija et al. 2008; Sunitha et al. 2008; Anantharaju and Muthiah 2008; Sharma et al. 2009).

Total soluble sugars showed a significant negative correlation with larval period, and positive correlation with pupation and pupal weight, while tannins showed a positive correlation with larval weight, pupation and adult emergence. From these observations, it was evident that, higher concentrations of these components favored better survival and development of $H$. armigera, leading to increased susceptibility of host plant to this pest. Low sugar and high phenol content has also been recorded in the resistant cultivars of pigeonpea against $H$. armigera (Sahoo and Patnaik 2003; Sharma et al. 2009). On the contrary, it is well established that tannins act as feeding deterrents and reduce the survival and development of insects (Bernards and Bastrup-Spohr 2008; Sharma et al. 2009) by precipitating proteins nonspecifically, thereby, inhibiting the digestion process (Bernards and Bastrup-Spohr 2008). However, protein binding activities of tannins depend on their chemical structure and other factors such as $\mathrm{pH}$ of the gut and concentration of antioxidants (Galati et al. 2002; Hagerman et al. 2003). Tannins not only act as feeding deterrents on the non-adapted insects, but also act as feeding stimulants on the adapted insects (Schultz 1989). Insects exhibit differential response to tannins of the host plant, based on their adoption capabilities to polyphenols (Barbenhenn et al. 2003). Protein and carbohydrate ratio also influences the toxicity of secondary metabolites (Raubenheimer 1992; Simpson and Raubenheimer 2001). While feeding on the plants, the herbivores ingest not only tannins, but also all other biomolecules in the host plant that effect their survival and development. The biological effects of tannins depend on their concentration and structure (Salminen and Karonen 2011).

There were significant differences among the genotypes tested with respect to flavonoid composition. The flavonoid compounds such as ferulic acid, naringin, 3,4-dihydroxy flavones and naringen were completely absent in cultivated chickpea, but were present only in a few wild relatives of chickpea. Wild relatives of chickpea had higher concentration of flavonoids as compared to the cultivated chickpea. Among the wild relatives, $C$. bijugum genotypes had higher concentration than in other species. More numbers of flavonoid compounds was present in IG 70012 and PI 599066 (C. bijugum) than in other genotypes. The flavonoid compounds exhibited negative correlations with larval survival, pupation, adult emergence and fecundity, whereas positive correlations were observed with the larval and pupal periods, which resulted in negative effects on survival and development of $H$. armigera. The flavonoid components present in wild relatives of chickpea such as judaicin 7-o-glucoside, 2-methoxy judaicin, judaicin and maakiain showed antifeedant activity, resulting in reduced larval weights of $H$. armigera (Simmonds and Stevenson 2001). Induction of flavonoids such as chlorogenic acid, syringic acid, quercetin, caffeic acid, vanillic acid and ferulic acid was greater in the resistant genotypes of groundnut in response to damage by $H$. armigera (War et al. 2016).The negative effects of flavonoids on insect performance in terms of prolonged developmental period, increased mortality, decreased survival, weight and 
fecundity had also been observed in many insect species, including Acyrthosiphon pisum Harris (Goławska et al. 2014), Epirrita autumnata Borkhausen (Lahtinen et al. 2004; Valkama et al. 2005), Mamestra configurata Walker (Onyilagha et al. 2004), Trichoplusia ni Hubner (Beninger et al. 2004), Nipaecoccus viridis Newstead (Lahtinen et al. 2006) and Eriosoma lanigerum Hausmann (Atteyat et al. 2012). The defense compounds of the host plant could act either by decreasing consumption and digestion or by acting as toxins (Scriber and Slansky 1981). Chlorogenic acid binds to the free amino acids and proteins leading to reduction in digestibility of plant tissue (Felton et al. 1992). Caffeic acid andchlorogenic acid caused protein oxidation leading to increased gut toxicity in herbivores (Summers and Felton 1994). The digestive enzyme activities were low in the midgut of $H$. armigera larvae when reared on diet treated with flavonoids such as chlorogenic acid, caffeic acid and protocatechuic acid (War et al. 2013). Though there are several reports on negative effects of flavonoids on the host plant, the exact mechanism by which flavonoids modulate the behavior of insects remains unknown (Simmonds 2003).

Reduced larval survival and weights leads to delay in developmental period due to secondary metabolites or poor nutritional quality. The changes in insect feeding behavior and poor food digestion lead to smaller pupae and reduced fecundity. The slower growth and prolonged development of larvae may also increase the probability of the larvae being subjected to predation and/or parasitism. Hence, the secondary metabolites of the host plant also play an important role in tritrophic interactions (Van Emden 1987). The wild relatives of chickpea with higher levels of antibiosis could be used as diverse sources of resistance to develop cultivars with stable resistance to $H$. armigera for sustainable crop production.

Acknowledgements We thank the entomology staff at International Crops Research Institute for the Semi-Arid Tropics (ICRISAT), Patancheru, India for their support in carrying out the experiments. The financial support provided by Department of Science and Technology (DST), New Delhi, India, under the INSPIRE Fellowship scheme to Dr Siva Kumar is gratefully acknowledged.

Funding This study was not funded by any organization.

\section{Compliance with ethical standards}

Conflict of interest The authors declare that they have no conflict of interest.

Ethical approval This article does not contain any studies with human participants or animals performed by any of the authors.

Informed consent This is not applicable as this article does not contain any studies with human participants performed by any of the authors.

\section{References}

Anantharaju P, Muthiah AR (2008) Biochemical components inrelation to pests incidence of pigeonpea spotted pod borer (Marucavitrata) and blister beetle (Mylabris spp.). Legume Res 31:87-93

Atteyat M, Abu-Romann S, Abu-Darwish M, Ghabeish I (2012) Impact of flavonoids against woolly apple aphid, Eriosoma lanigerum (Hausmann) and its sole parasitoid, Aphelinus mali (Hald.). J Agric Sci 4:227-236

Babu GC, Sharma HC, Madhumati T, Raghavaiah G, Murthy KVMK, Rao VS (2014) A semi-synthetic chickpea flour based diet for long-term maintenance of laboratory culture of Helicoverpa armigera. Indian J Entomol 76:336-340

Ballhorn DJ, Kautz S, Jensen M, Schmitt I, Heil M, Hegeman AD (2011) Genetic and environmental interactions determine plant defenses against herbivores. Ecology 99:313-326

Banu MR, Muthiah AR, Ashok S (2005) Evaluation of pigeonpea (Cajanus cajan L.) genotypes against gram-pod borer (Helicoverpa armigera). Abstract in 4th international food legume research conference on food Legumes for nutritional security and sustainable agriculture, New Delhi, India, p 317

Barbenhenn RV, Poopat U, Spencer B (2003) Semiquinone and ascorbyl radicals in the gut fluids of caterpillars measured with EPR spectrometry. Insect Biochem Mol Biol 33:125-130

Behmer ST (2008) Insect herbivore nutrient regulation. Annu Rev Entomol 54:165

Beninger CW, Abou-Zaid MM, Kistner ALE, Hallett RH, Iqbal MJ, Grodzinski B, Hall JC (2004) A flavanone and two phenolic acids from Chrysanthemum morifolium with phytotoxic and insect growth regulating activity. J Chem Ecol 30:589-606

Bennett RN, Wallsgrove RM (1994) Secondary metabolites in plant defence mechanisms. New Phytol 127:617-633

Bernards MA, Bastrup-Spohr L (2008) Phenylpropanoid metabolism induced by wounding and insect herbivory. In: Schaller A (ed) Induced plant resistance to herbivory. Springer, Berlin, pp 189-211

Bhonwong A, Stout MJ, Attajarusit J, Tantasawat P (2009) Defensive role of tomato polyhenol oxidase against cotton bollworm (Helicoverpa armigera) and beet armyworm (Spodoptera exigua). J Chem Ecol 35:28-38

Blanco-Labra A, Chagolla-Lopez A, Martinez-Gallardo N, ValdesRodriguez S (1995) Further characterization of the $12 \mathrm{kDa}$ protease/alpha amylase inhibitor present in maize seeds. J Food Biochem 19:27-41

Bray HC, Thorpe WV (1954) Analysis of phenolic compounds of interest in metabolism. Methods Biochem Anal 1:27-52

Burns RE (1971) Method for estimation of tannin in grain sorghum. Agron J 63(3):511-512

Cates RG (1980) Feeding patterns of monophagous, oligophagous, and polyphagous insectherbivores: the effect of resource abundance and plant chemistry. Oecologia 46(1):22-31

Dakora FD, Phillips DA (1996) Diverse functions of isoflavonoids in legumes transcend anti-microbial definitions of phytoalexins. Physiol Mol Plant Pathol 49:1-20

FAO STAT (2016) Food and Agriculture Organisation of the United Nations 2002. IOP Publishing FAO. https://apps.fao.org. Accessed 9 Mar 2018

Felton GW, Donato KK, Broadway RM, Duffey SS (1992) Impact ofoxidized plant phenolics on the nutritional quality of dietary protein to a noctuid herbivore, Spodoptera exigua. J Insect Physiol 38:277-285

Galati G, Sabzevari O, Wilson JX, O’Brien PJ (2002) Prooxidant activity and cellular effects of the phenoxyl radicals of dietary flavonoids and other polyphenolics. Toxicology 177:91-104 
Giri AP, Harsulkar AM, Deshpande VV, Sainani MN, Gupta VS, Ranjekar PK (1998) Chickpea defensive proteinase inhibitors can be inactivated by pod borer gut proteinases. Plant Physiol 116:393-401

Girija SPM, Patil SA, Gowda CLL, Sharma HC (2008) Biophysical and biochemical basis of host plant resistance to pod borer, Helicoverpa armigera (Hubner) in chickpea (Cicer arietinum L.). Indian J Genet Pl Br 68(3):320-323

Goławska S, Sprawka I, Łukasik I, Goławski A (2014) Are naringenin and quercetin useful chemicals in pest-management strategies? J Pest Sci 87:173-180

Golla SK, Rajasekhar P, Sharma SP, HariPrasad KV, Sharma HC (2018) Antixenosis and antibiosis mechanisms of resistance to pod borer, Helicoverpa armigera in wild relatives of chickpea, Cicer arietinum. Euphytica. https://doi.org/10.1007/s1068 1-018-2168-5

Hagerman AE, Dean RT, Davies MJ (2003) Radical chemistry of epigallocatechin gallate and its relevance to protein damage. Arch Biochem Biophys 414:115-120

Hahn DH, Faubion JM, Rooney LW (1983) Sorghum phenolic acids, their performance liquid chromatography separation and their relation to fungal resistance. Cereal Chem 60:255-259

Hedge JE, Hofreiter BT (1962) Anthrone determination for carbohydrate. In: Whistley RL, Be Miller JN (eds) Carbohydrate chemistry. Academic Press, New York, p 17

Kanchana R, Lakshmi KV, Rajasekhar P (2005) Morphological and biochemical bases of host plant resistance to Helicoverpa armigera (Hubner) in chickpea. J Plant Protect Environ 2(1):12-17

Kansal R, Kumar M, Kuhar K, Gupta RN, Subrahmanyam B, Koundal KR, Gupta VK (2008) Purification and characterization of trypsin inhibitor from Cicer arietinum (L.) and its efficacy against Helicoverpa armigera. Braz J Plant Physiol 20(4):313-322

Kaur R, Gupta AK, Taggar GK (2014) Role of catalase, $\mathrm{H}_{2} \mathrm{O}_{2}$ and phenolics in resistance of pigeonpea towards Helicoverpa armigera (Hubner). Acta Physiol Plant 36:1513-1527

Kennedy GG (2003) Tomato, pests, parasitoids and predators: tritrophic interactions involving the genus Lycopersicon. Annu Rev Entomol 48:51-72

Kranthi KR, Jadhav DR, Kranthi S, Wanjari RR, Ali SS, Russel DA (2002) Insecticide resistance in five major insect pests of cotton in India. Crop Prot 21:449-460

Lahtinen M, Salminen JP, Kapari L, Lempa K, Ossipov V, Sinkkonen J, Valkama E, Haukioja E, Pihlaja K (2004) Defensive effect surface flavonoid aglycones of Betula pubescens leaves against first instar Epirrita autumnata larvae. J Chem Ecol 30:2257-2268

Lahtinen M, Kapari L, Haukioja E, Pihlaja K (2006) Effects of increased content of leaf surface flavonoids on the performance of mountain birch feeding sawflies vary for early and late season species. Chemoecology 16:159-167

Lowry OH, Rosebrough NJ, Farr AL, Ramdall RJ (1951) Protein measurement with Folin phenol reagent. J Biol Chem 193:265-275

Maffei ME, Mithofer A, Boland W (2007) Insects feeding on plants: rapid signals and responses preceding the induction of phytochemical release. Phytochemistry 68:2946-2959

Musayimana T, Saxena RC, Kaimu EW, Ogol CPKO, Khan ZR (2001) Effects of neem seed derivatives on behavioral and physiological responses of the Cosmopolites sordidus (Coleoptera: Curculionidae). J Econ Entomol 94:449-454

Napal GND, Defago MT, Valladares GR, Palacios SM (2010) Response of Epilachna paenulata to two flavonoids, pinocembrin and quercetin, in a comparative study. J Chem Ecol 36(8):898-904

Narayanamma VL, Sharma HC, Gowda CLL, Sriramulu M (2008) Incorporation of lyophilized leaves and pods into artificial diets to assess the antibiosis component of resistance to pod borer, Helicoverpa armigera (Hubner) (Lepidoptera:Noctuidae) in chickpea. Int J Trop Insect Sc 27(3/4):191-198
Onyilagha JC, Lazorko J, Gruber MY, Soroka JJ, Erlandson MA (2004) Effect of flavonoids on feeding preference and development of the crucifer pest Mamestra configurata Walker. J Chem Ecol 30:109-124

Panzuto M, Mauffettes Y, Albert PJ (2002) Developmental, gustatory, and behavioral responses of leaf roller larvae, Choristoneura rosaceana, to tannic acid and glucose. J Chem Ecol 28:145-160

Parde VD, Sharma HC, Kachole MS (2012) Protease inhibitors in wild relatives of pigeonpea against the cotton bollworm/legume pod borer, Helicoverpa armigera. Am J Plant Sci 3:627-635

Patankar AG, Harsulkar AM, Giri AP, Gupta VS, Sainani MN, Ranjekar PK, Deshpande VV (1999) Diversity in inhibitors of trypsin and Helicoverpa armigera gut proteinases in chickpea (Cicer arietinum) and its wild relatives. Theor Appl Genet 99:719-726

Raubenheimer D (1992) Tannic acid, protein, and digestible carbohydrate: dietary imbalance and nutritional compensation in locusts. Ecology 73:1012-1027

Roeder KA, Behmer ST (2014) Lifetime consequences of food protein-carbohydrate content for an insect herbivore. Funct Ecol 28:1135-1143

Rupalighodeswar JR, Chavan JK (2003) Biochemical analysis of chickpea cultivars in relation to pod borer infestation. Indian J Agric Biochem 1691:47-48

Sahoo BK, Patnaik HP (2003) Effect of biochemicals on the incidence of pigeonpea pod borers. Indian J Plant Prot 31(1):105-1081

Saini HS, Weder JKP, Knights EJ (1992) Inhibitor activities of chickpeas (Cicer arietinum L.) against bovine, porcine and human trypsin and chymotrypsin. J Sci Food Agric 60:287-295

Salminen JP, Karonen M (2011) Chemical ecology of tannins and other phenolics: we need a change in approach. Funct Ecol 25:325-338

Sarode SV (1999) Sustainable management of Helicoverpa armigera (Hubner). Pestology 13(2):279-284

Schultz JC (1989) Tannin-insect interactions. In: Hemingway RW, Karchesy JJ (eds) Chemistry and significance of condensed tannins. Plenum Press, NewYork, pp 417-433

Scriber JM, Slansky F Jr (1981) The nutritional ecology of immature insects. Annu Rev Entomol 26:183-211

Selvanarayanan V, Narayanasamy P (2006) Assessment of tomato germplasm for resistance to fruit borer Helicoverpa armigera (Hubner). J Veg Sci 12:71-79

Shanower TG, Yoshida M, Peter AJ (1997) Survival, growth, fecundity, and behavior of Helicoverpa armigera (Lepidoptera:Noctuidae) on pigeonpea and two wild Cajanus species. J Econ Entomol 90(3):837-841

Sharma HC (2001) Cotton bollworm/legume pod borer, Helicoverpa armigera (Hubner) (Noctuidae:Lepidoptera) biology and management. In: Crop protection compendium. CAB International, Wallingford

Sharma HC, Pampapathy G, Lanka SK, Ridsdill-Smith TJ (2005a) Antibiosis mechanism of resistant to pod borer, Helicoverpa armigera in wild relatives of chickpea. Euphytica 142:107-117

Sharma HC, Pampapathy G, Lanka SK, Ridsdill-Smith TJ (2005b) Exploitation of wild Cicer reticulatum germplasm for resistance to Helicoverpa armigera. J Econ Entomol 98(6):2246-2253

Sharma HC, Bhagawat MP, Pampapathy G, Sharma JP, Ridsdill-Smith TJ (2006) Perennial wild relatives of chickpea as potential sources of resistance to Helicoverpa armigera. Genet Resour Crop Evol 53:131-138

Sharma HC, Sujana G, Rao DM (2009) Morphological and chemical components of resistance to pod borer, Helicoverpa armigerain wild relatives of pigeonpea. Arthropod-Plant Interact 3:151-161

Simmonds MSJ (2003) Flavonoid-insect interactions: recent advances in our knowledge. Phytochemistry 6:21-30

Simmonds MSJ, Stevenson PC (2001) Effects of isoflavonoid from Cicer arietinum on larvae of Helicoverpa armigera. J Chem Ecol 27(5):965-977 
Simpson SJ, Raubenheimer D (2001) The geometric analysis of nutrient allelochemical interactions: a case study using locusts. Ecology 82:422-439

Simpson SJ, Raubenheimer D (2009) Macronutrient balance and lifespan. Aging 1:875

Sujana G, Sharma HC, Rao DM (2008) Antixenosis and antibiosis components of resistance to pod borer Helicoverpa armigera in wild relatives of pigeonpea. Int J Trop Insect Sci 28(4):191-200

Summers CB, Felton GW (1994) Prooxidant effects of phenolic acidson the generalist herbivore Helicoverpa zea (Lepidoptera:Noctuidae): potential mode of action for phenolic compoundsin plant antiherbivore chemistry. Insect Biochem Mol Biol 24:943-953

Sunitha V, Rao GVR, Lakshmi KV, Saxena KB, RameshwarRao V, Reddy YVR (2008) Morphological and biochemical factors associated with resistance to Maruca vitrata (Lepidoptera:Pyralidae) in short-duration pigeonpea. Int J Trop Insect Sci 28:45-52

Treutter D (2006) Significance of flavonoids in plantresistance: a review. Environ Chem Lett 4:147-157

Udamale SK, Moharil MP, Ugale TB, Mankar JM (2013) Differential inhibition of Helicoverpa armigera (Hubner) gut proteinases by proteinase inhibitors of okra and its wild relatives. ISRN Biotechnol 632173:1-10
Valkama E, Koricheva J, Salminen J-P, Helander M, Saloniemi I, Saikkonen K, Pihlaja K (2005) Leaf surface traits: over looked determinants of birch resistance to herbivores and foliar micro fungi? Trees 19:191-197

Van Emden HF (1987) Cultural methods: the plant. In: Burn AJ, Coaker TH, Jepson PC (eds) Integrated pest management. Academic Press, London, pp 27-68

Walling LL (2000) The myriad plant responses to herbivores. J Plant Growth Regul 19:195-216

War AR, Paulraj MG, Hussain B, Buhroo AA, Ignacimuthu A, Sharma HC (2013) Effect of plant secondary metabolites on legume pod borer, Helicoverpa armigera. J Pest Sci 86:399-408

War AR, Sharma S, Sharma HC (2016) Differential induction of flavonoids in groundnut in response to Helicoverpa armigera and Aphis craccivora infestation. Int J Insect Sci 8:55-64

Publisher's Note Springer Nature remains neutral with regard to jurisdictional claims in published maps and institutional affiliations. 